\title{
LECTURES ON MARKED LENGTH SPECTRUM RIGIDITY (PRELIMINARY VERSION)
}

\author{
A. WILKINSON
}

\section{INTRODUCTION}

Let $M$ be a closed Riemannian manifold whose sectional curvatures are all negative, and denote by $\mathcal{C}$ the set of free homotopy classes of closed curves in $M$. Negative curvature implies that in each free homotopy class, there is a unique closed geodesic. This defines a marked length spectrum function $\ell: \mathcal{C} \rightarrow \mathbb{R}_{>0}$ which assigns to the class $g$ the length $\ell(g)$ of this closed geodesic. Burns and Katok asked whether the function $\ell$ determines $M$, up to isometry [7]. This question remains open in general, but has been solved completely for surfaces by Otal [27] and independently slightly later, but in greater generality by Croke [8].

In these notes, I'll explain in several steps a proof of this marked length spectrum rigidity for negatively curved surfaces:

Theorem 0.1 (Otal). Let $S$ and $S^{\prime}$ be closed, negatively curved surfaces with the same marked length spectrum. Then $S$ is isometric to $S^{\prime}$.

Many of the ideas I'll present fit neatly into the broader contexts of nonpositively curved manifolds and even hyperbolic groups. See [15] for a dictionary between some of the concepts discussed here and analogous questions in geometric group theory.

The overall focus of my presentation will be on the interplay between geometry and smooth dynamics. While I will try to keep the lectures on geometric footing, these notes give a more thorough discussion of the dynamics of geodesic flows, and the exercises should help build some dynamical intuition. Most of the material discussed here can be regarded as an introduction to the foundations of geodesic flows in nonpositive curvature, boundaries of Hadamard spaces, and the dynamics of boundary actions of isometry groups.

The exposition is deliberately inefficient: several overlapping concepts from a variety of sources are introduced, and I have tried to illuminate the precise connections between them. While these concepts duplicate each other in this context, they independently generalize into other contexts and the connections between them can help build intuition. The notes here are a significantly expanded version of a 4-lecture course given at the Park City Math Institute (PCMI) July 9-13, 2012.

Date: December 11, 2012. 
Remark: The (unmarked) length spectrum is defined to be the set of lengths $\{\ell(g): g \in \mathcal{C}\}$, counted with multiplicity . The length spectrum does not determine the manifold up to isometry. Examples exist even for surfaces of constant negative curvature $[32,30]$.

Remark: There is a connection between length spectrum and spectrum of the Laplacian. On hyperbolic manifolds, the Selberg trace formula shows that the spectrum of the Laplacian determines the length spectrum. For generic Riemannian metrics, the spectrum of the Laplacian determines the length spectrum. The analogous spectral rigidity question for the spectrum of the Laplacian was posed by Kac. Such rigidity does not hold in general (one cannot "hear the shape of a drum") but does hold along deformations of negatively curved metrics $[17,11]$. See $[16,31]$ for a discussion of these and related rigidity problems.

Thanks to Alex Wright and Curt McMullen for very useful discussions.

\section{LECTURE 1}

1.1. Background on negatively curved surfaces. Let $S$ be a compact, negatively curved surface, and let $\tilde{S}$ be its universal cover. Since $S$ is a surface, all notions of curvature coincide (sectional, Gaussian, Ricci...), and the curvature can thus be expressed as a function $k: S \rightarrow \mathbb{R}_{<0}$ which pulls back to a bounded function $k: \tilde{S} \rightarrow \mathbb{R}_{<0}$. The Riemann structure defines a Levi-Civita connection $\nabla$, which in turn defines notions of covariant differentiation and parallel translation. A vector field $X$ along a curve $c(t)$ is parallel if $\nabla_{\dot{c}(t)} X(c(t))=0$ for all $t$.

A curve $\gamma$ is a geodesic if its velocity curve is parallel along itself:

$$
\nabla_{\dot{\gamma}} \dot{\gamma} \equiv 0
$$

Regarded in local coordinates, equation (1) is a second-order ODE. A tangent vector $v \in T \tilde{S}$ supplies an initial value:

$$
\dot{\gamma}(0)=v .
$$

Since the connection is $C^{\infty}$, the initial value problem given by (1) and (2) has a unique solution. Because the Riemann structure on $\tilde{S}$ is the pullback of a structure on a compact manifold, this solution is defined for all time. For a tangent vector $v \in T \tilde{S}$, we denote by $\gamma_{v}:(-\infty, \infty) \rightarrow \tilde{S}$ this unique geodesic with $\dot{\gamma}_{v}(0)=v$. (For background on the geodesic equation see, e.g. $[22])$. Solutions to ODEs depend smoothly on parameters, so the map

$$
(t, v) \mapsto \gamma_{v}(t)
$$

from $\mathbb{R} \times T \tilde{S} \rightarrow \tilde{S}$ is $C^{\infty}$. As parallel transport preserves the Riemann structure, the speed $\left\|\dot{\gamma}_{v}(t)\right\|$ is constant, equal to $\|v\|$. One therefore obtains a 1-1 correspondence between unit-speed geodesics and the unit tangent bundle $T^{1} \tilde{S}$ given by $v \leftrightarrow \gamma_{v}$. 


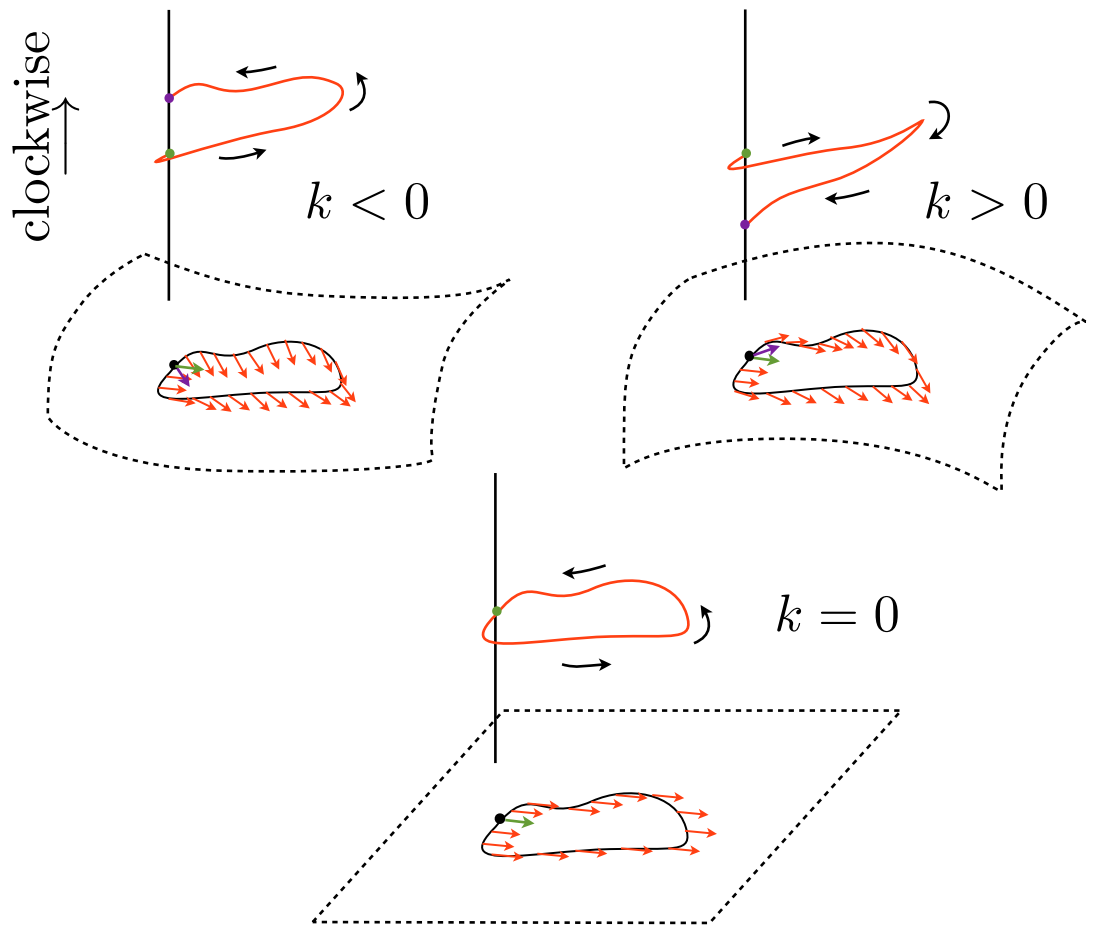

Figure 1. Parallel transport in negative, positive and zero curvature. The vertical line in each diagram represents the fiber of the unit tangent bundle, and the red curve represents the parallel lift of the back curve below. The change in angle as the green vector is parallel translated in a counterclockwise fashion around the black curve results in a vertical jump in the fiber: clockwise in negative curvature, counterclockwise in positive curvature, and no change for 0 curvature.

The Cartan-Hadamard theorem states in this setting that for any $p \in \tilde{S}$, the exponential map

$$
\exp _{p}: w \in T_{p} \tilde{S} \mapsto \gamma_{w}(1)
$$

is a $C^{\infty}$ diffeomorphism onto $\tilde{S}$. Consequently, $\tilde{S}$ is contractible, diffeomorphic to the plane $\mathbb{R}^{2}$.

1.2. A key example. A key example is the hyperbolic plane. The Poincaré disk (or hyperbolic disk) is the domain $\mathbb{D}=\{z:|z|<1\}$ with the metric

$$
d s^{2}=\frac{4|d z|^{2}}{\left(1-|z|^{2}\right)^{2}} .
$$

The group of orientation-preserving isometries of $\mathbb{D}$ is

$$
\left\{\left(\begin{array}{cc}
\alpha & \beta \\
\bar{\beta} & \bar{\alpha}
\end{array}\right):|\alpha|^{2}-|\beta|^{2} \neq 0\right\}
$$


which acts by Möbius transformations:

$$
\left(\begin{array}{cc}
\alpha & \beta \\
\bar{\beta} & \bar{\alpha}
\end{array}\right): z \mapsto \frac{\alpha z+\beta}{\bar{\beta} z+\bar{\alpha}} .
$$

The hyperbolic disk is isometric via a Möbius transformation to the upperhalf plane $\mathbb{H}=\operatorname{Im}(z)>0$ with the metric

$$
d s^{2}=\frac{|d z|^{2}}{(\operatorname{Im} z)^{2}} .
$$

The isometry group of $\mathbb{H}$ is

$$
\operatorname{PSL}(2, \mathbb{R})=\left\{\left(\begin{array}{ll}
a & b \\
c & d
\end{array}\right): a d-b c=1\right\} /\{ \pm I\},
$$

also acting by Möbius transformations. The curvature of $\mathbb{H}$ is constant, equal to -1 . We will refer to the $\mathbb{D}$ and $\mathbb{H}$ models interchangeably.

Hyperbolic geodesics in $\mathbb{D}$ are Euclidean circular arcs, perpendicular to $\partial \mathbb{D}=\{|z|=1\}$. In $\mathbb{H}$, hyperbolic geodesics in $\mathbb{H}$ are Euclidean (semi) circular arcs, perpendicular to $\operatorname{Im}(z)=0$ (where lines are Euclidean circles with infinite radius).

The stabilizer of a point under this left action is the compact subgroup $K=S O(2) /\{ \pm I\}$, which gives an identification of $\mathbb{H}$ with the coset space of $K$ :

$$
\mathbb{H}=\operatorname{PSL}(2, \mathbb{R}) / K .
$$

The derivative action of $P S L(2, \mathbb{R})$ on the unit tangent bundle $T^{1} \mathbb{H}$ is free and transitive, and gives an analytic identification between $T^{1} \mathbb{H}$ and $P S L(2, \mathbb{R})$. The action of $P S L(2, \mathbb{R})$ on $T^{1} \mathbb{H}$ by isometries corresponds to left multiplication in $\operatorname{PSL}(2, \mathbb{R})$.

If $S$ is a closed orientable surface with $\tilde{S}=\mathbb{H}$, then $\pi_{1}(S)$ acts by isometries on $\mathbb{H}$ and hence embeds as a discrete subgroup $\Gamma<\operatorname{PSL}(2, \mathbb{R})$. We thus obtain the following identifications:

$$
S=\Gamma \backslash \mathbb{H}=\Gamma \backslash \operatorname{PSL}(2, \mathbb{R}) / K,
$$

and

$$
T^{1} S=\Gamma \backslash \operatorname{PSL}(2, \mathbb{R}) .
$$

Endowing $\operatorname{PSL}(2, \mathbb{R})$ with a suitable left-invariant metric gives an isometry between $\operatorname{PSL}(2, \mathbb{R}) / K$ and $\mathbb{H}$. This metric on $\operatorname{PSL}(2, \mathbb{R})$ also induces a metric on $T^{1} \mathbb{H}$, called the Sasaki metric (see the next section). In this metric, the lifts of geodesics in $\mathbb{H}$ via $\gamma \mapsto \dot{\gamma}$ gives Sasaki geodesics in $T^{1} \mathbb{H}$ (there are other Sasaki geodesics that do not project to geodesics in $\mathbb{H}$ but project to curves of constant geodesic curvature: for example, the orbits of the $S O(2)$ subgroup.)

Exercise 1.1. If you have never done so before, verify these assertions about hyperbolic space. Useful fact: the curvature of a conformal metric 
$d s^{2}=h(z)^{2}|d z|^{2}$ (where $h$ is real-valued and positive) on a planar domain is given by the formula:

$$
k=-\frac{\Delta \log h}{h^{2}},
$$

where $\Delta$ is the Euclidean Laplacian.

To verify the assertion about geodesics, it suffices to show that the curve $t \mapsto i e^{t}$ is a geodesic in $\mathbb{H}$ and then apply isometries. (note that this vertical ray in $\mathbb{H}$ is fixed pointwise by the (orientation-reversing) hyperbolic isometry $z \mapsto-\bar{z} \ldots)$. One can also find a formula for hyperbolic distance using this method.

To identify $T^{1} \mathbb{H}$ with $\operatorname{PSL}(2, \mathbb{R})$, start by identifying the unit vertical tangent vector based at $i$ with the identity matrix. It is helpful to understand the orbit of this vector under one-parameter subgroups that together generate $\operatorname{PSL}(2, \mathbb{R})$, for example, the groups in the Iwasawa (KAN) decomposition.

Any closed orientable surface of genus 2 or higher admits a metric of constant negative curvature. There are a variety of methods to construct such a metric. One way is to find a discrete and faithful representation $\rho: \pi_{1}(S) \rightarrow \operatorname{PSL}(2, \mathbb{R})$, and set

$$
S=\Gamma \backslash \operatorname{PSL}(2, \mathbb{R}) / K
$$

as above, with $\Gamma=\rho\left(\pi_{1}(S)\right)$. Using algebraic methods, one can find arithmetic subgroups (and so arithmetic surfaces) in this way.

A highly symmetric, hands-on way to construct a genus $g$ surface is to take a regular hyperbolic $4 g$-gon with sum of vertex angles equal to $2 \pi$ and use hyperbolic isometries to glue opposite sides. Much more generally, hyperbolic structures are constructed by gluing together $2 g$ hyperbolic "pairs of pants" of varying cuff lengths. There are $6 g-6$ degrees of freedom in this construction (cuff lengths of pants and twist parameters in gluing). The space of all such structures is a $6 g-6$-dimensional space diffeomorphic to a ball called Teichmüller space, and the space of of such structures modulo isometry is a $6 g-6$ dimensional orbiford called Moduli space.

1.3. Geodesics in negative curvature. In the sequel, $S$ is a closed, orientable negatively curved surface. Henceforth, all geodesics are unit speed, unless otherwise specified. To fix concepts, we endow the tangent bundle $T S$ with a fixed Riemann structure called the Sasaki metric, which is compatible with the negatively curved structure on $S .{ }^{1}$ This pulls back to a Riemann structure on $T^{1} \tilde{S}$. In what follows, the distances $d_{T^{1} S}$ and $d_{T^{1} \tilde{S}}$ on $T^{1} S$ and $T^{1} \tilde{S}$ are measured in this Sasaki metric. This metric has the property that its restriction to any fiber of $T^{1} S$ is just Lebesgue (angular) measure $d \theta$, and its restriction to any parallel vector field along a curve in

\footnotetext{
${ }^{1}$ Briefly, the Sasaki structure in the tangent space $T_{v} T S$ to $v \in T S$ is obtained using the identification $T_{v}(T S) \simeq T_{\pi(v)} S \times T_{\pi(v)} S$ given by the connection. The two factors are endowed with the original Riemann inner product and declared to be orthogonal. See, e.g. [22]).
} 
$S$ is just arclength along that curve. On the hyperbolic plane, the Sasaki metric is precisely the left-invariant metric on $\operatorname{PSL}(2, \mathbb{R})$.

For geodesics $\gamma_{1}, \gamma_{2}$, we write $\gamma_{1} \sim \gamma_{2}$ if one is an orientation-preserving reparamentrization of the other: $\gamma_{2}(t)=\gamma_{1}\left(t+t_{0}\right)$, for some $t_{0} \in \mathbb{R}$. Denote by $[\gamma]$ the equivalence class of the parametrized unit speed geodesic $\gamma$.
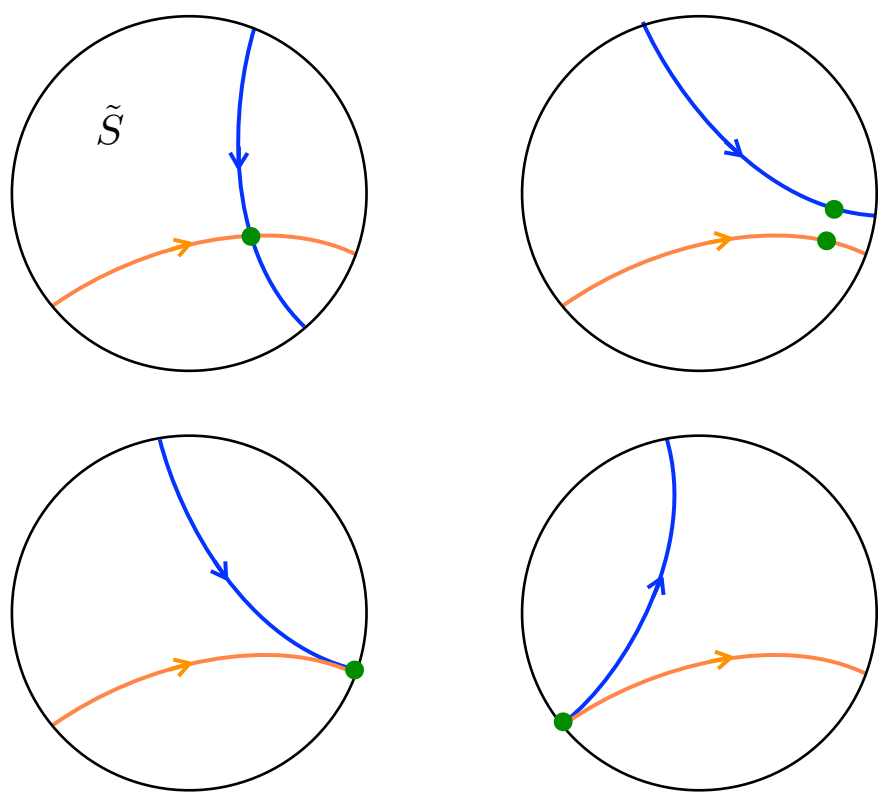

FIGURE 2. Geodesics in negative curvature. We have marked the points where distance between the distinct geodesics is minimized. Clockwise from top left: intersecting geodesics; nonintersecting, non asymptotic geodesics; backward asymptotic geodesics; and forward asymptotic geodesics.

Proposition 1.2. The universal cover $\tilde{S}$ has the following properties:

(1) Strict Convexity: If $\gamma_{1}, \gamma_{2}$ are distinct unit speed geodesics, then

$$
t \mapsto d\left(\gamma_{1}(t), \gamma_{2}(\mathbb{R})\right) \quad \text { and } \quad t \mapsto d_{T^{1} \tilde{S}}\left(\dot{\gamma}_{1}(t), \dot{\gamma}_{2}(\mathbb{R})\right)
$$

are strictly convex functions acheiving their minimum at the same time $t_{0}$ (with the possibility $t_{0} \in\{ \pm \infty\}$ ).

Thus the distance between two unit speed geodesics is realized at a unique point, where the (Sasaki) distance between velocity curves is also minimized (including the possibility that the geodesics are asymptotic at either $+\infty$ or $-\infty$ ). 
(2) Geodesic rays are asymptotic or diverge: If $\gamma_{1}, \gamma_{2}:[0, \infty) \rightarrow \tilde{S}$ are geodesic rays with

$$
\limsup _{t \rightarrow \infty} d\left(\gamma_{1}(t), \gamma_{2}(t)\right)<\infty
$$

then there exists $t_{0} \in \mathbb{R}$ such that

$$
\lim _{t \rightarrow \infty} d\left(\gamma_{1}(t), \gamma_{2}\left(t+t_{0}\right)\right)=0
$$

(in fact, this convergence to 0 is uniformly exponentially fast, with rate determined by the curvature $k$ ).

(3) Distinct geodesics diverge: For every $C, \epsilon>0$, there exists $T>0$ such that, for any two unit speed geodesics: $\gamma_{1}, \gamma_{2}:(-\infty, \infty) \rightarrow \tilde{S}$, if

$$
\max \left\{d\left(\gamma_{1}(-T), d\left(\gamma_{2}(-T)\right), d\left(\gamma_{1}(T), \gamma_{2}(T)\right)\right\}<C,\right.
$$

then

$$
d_{T^{1} \tilde{S}}\left(\dot{\gamma}_{1}(0), \dot{\gamma}_{2}([-T, T])\right)<\epsilon .
$$

In particular, if

$$
d\left(\gamma_{1}(t), \gamma_{2}(t)\right)<C
$$

for all $t$, then $\gamma_{1} \sim \gamma_{2}$.

1.4. The geodesic flow. The geodesic flow $\varphi: T \tilde{S} \times \mathbb{R} \rightarrow T \tilde{S}$ sends $(v, t)$ to $\varphi_{t}(v):=\dot{\gamma}_{v}(t)$. As remarked above, the dependence of $\dot{\gamma}_{v}(t)$ on $v$ and $t$ is $C^{\infty}$, and uniqueness of solutions to the initial value problem (1) and (2) implies that $\varphi_{t}$ is a flow on $T^{1} \tilde{S}$, i.e. a 1-parameter group of diffeomorphisms under composition:

$$
\varphi_{0}=I d, \quad \text { and } \varphi_{s+t}=\varphi_{s} \circ \varphi_{t}, \quad \forall s, t .
$$

This projects to a geodesic flow on $T S$, since the geodesic flow commutes with isometries. Since geodesics have constant speed, the geodesic flow restricts to a geodesic flow on the unit tangent bundles $T^{1} S, T^{1} \tilde{S}$.

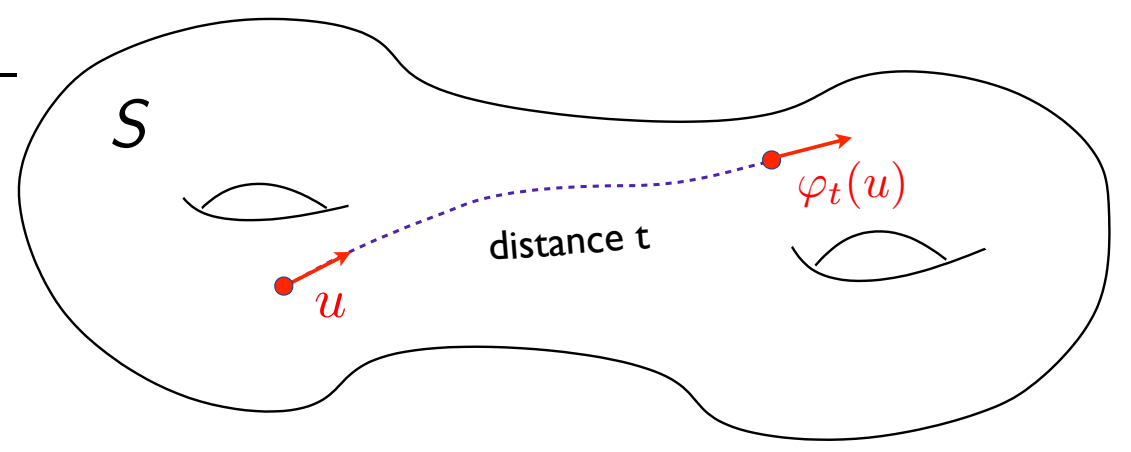

Figure 3. The geodesic flow for a surface $S$. 
Exercise 1.3. An additional symmetry of the geodesic flow is flip invariance:

$$
\varphi_{-t}(-v)=-\varphi_{t}(v) .
$$

Another way to state this is that $\varphi_{t}$ is conjugate to the reverse time flow $\varphi_{-t}$ via the involution on $I: T^{1} \tilde{S} \rightarrow T^{1} S$ defined by:

$$
I(v)=-v \text {. }
$$

Verify this.

Another useful way to describe the geodesic flow is as the flow of a Hamiltonian vector field on $T S$ (and similarly on $T \tilde{S}$ ). To do this, one first recalls that the cotangent bundle $T^{*} S$ carries a canonical symplectic structure (it is $\omega=d \theta$, where $\theta$ is the canonical 1 -form on $T^{*} S$ ). This pulls back to a (noncanonical) symplectic form $\omega$ on the tangent bundle via the Riemann structure. Let $E: T S \rightarrow \mathbb{R}$ be the Hamiltonian (energy) function given by the half the square of the Riemannian metric:

$$
E(v)=\frac{1}{2}\|v\|^{2}
$$

Then the symplectic gradient $X_{E}$ of this Hamiltonian is defined by:

$$
d E=i_{X_{E}} \omega
$$

The vector field $X_{E}$ on $T S$ then generates the geodesic flow; i.e.,

$$
\left.\frac{d}{d t} \varphi_{t}(v)\right|_{t=t_{0}}=X_{E}\left(\varphi_{t_{0}}(v)\right)
$$

for all $v, t_{0}$ (this is just another formulation of the geodesic equation given by (1) and (2)).

From standard properties of Hamiltonian flows and the sympectic structure on the (co)tangent bundle $T S$, one reads off immediately properties of the geodesic flow:

(1) $E \circ \varphi_{t}=E$, for all $t$. That is, the geodesic flow preserves length.

(2) $\varphi_{t}^{*} \omega=\omega$, for all $t$. That is, $\left\{\varphi_{t}\right\}$ is a 1-parameter group of symplectomorphisms. In particular, $\varphi_{t}$ preserves the volume form $\omega \wedge \omega$.

(3) The restriction of $\varphi_{t}$ to the unit tangent bundle $T^{1} \tilde{S}=E^{-1}(1)$ preserves the contact 1 -form $\alpha$ given by ${ }^{2}$

$$
\alpha=i_{\nabla E} \omega .
$$

In particular, $\varphi_{t}$ preserves the volume form $d \lambda=\alpha \wedge d \alpha$ on $T^{1} S$ (Liouville's Theorem). This volume $\lambda$ is called the Liouville measure. It is the product of Riemannian measure on $S$ with arclength on the fibers of $T^{1} S$.

\footnotetext{
${ }^{2}$ The gradient $\nabla E$ in this formula is defined with respect to the Sasaki metric on $T S$. Alternately, the 1-form $\alpha$ can be defined as the pullback of the canonical 1-form $\theta$ on the cotangent bundle $T^{*} S$ under the isomorphism between $T S$ and $T^{*} S$ induced by the given Riemann structure on $S$.
} 
(4) Since $T^{1} S$ is compact, its total volume is finite:

$$
\lambda\left(T^{1} S\right)=\int_{T^{1} S}|\alpha \wedge d \alpha|<\infty .
$$

Poincaré Recurrence implies that for almost every $v \in T^{1} S$ (with respect to volume):

$$
\liminf _{t \rightarrow \pm \infty} d_{T^{1} S}\left(\varphi_{t}(v), v\right)=0 .
$$

More generally, the machinery of smooth ergodic theory can be applied to geodesic flows to prove things like ergodicity, mixing etc.

(See [21] for more details. Some of what is written here is completely general and applies to any Riemannian manifold.)

Exercise 1.4. Show that on $T^{1} \mathbb{H}=P S L(2, \mathbb{R})$, the geodesic flow is given by right multiplication by the 1-parameter subgroup:

$$
A=\left\{a_{t}:=\left(\begin{array}{cc}
e^{t / 2} & 0 \\
0 & e^{-t / 2}
\end{array}\right): t \in \mathbb{R}\right\}
$$

\section{LECTURE 2}

2.1. Busemann functions and horospheres. Given a unit speed geodesic $\gamma$, we define the (forward and backward) Busemann functions

$$
b_{\gamma}^{+}, b_{\gamma}^{-}: \tilde{S} \rightarrow \mathbb{R}
$$

by

$$
b_{\gamma}^{+}(p)=\lim _{t \rightarrow \infty} d(\gamma(t), p)-t
$$

and

$$
b_{\gamma}^{-}(v)=\lim _{t \rightarrow \infty} d(\gamma(-t), p)-t
$$

These limits always exist by Proposition 1.2, part (2), and are finite. Under the identification of unit-speed (parametrized) geodesics with $T^{1} \tilde{S}$, we write $b_{v}^{ \pm}$for $b_{\gamma_{v}}^{ \pm}$.

Exercise 2.1. Prove the following:

(1) $b_{\varphi_{t}(v)}^{ \pm}=b_{v}^{ \pm}-t$,

(2) $\gamma_{1} \sim \gamma_{2}$ if and only if $\left|b_{\gamma_{1}}^{ \pm}-b_{\gamma_{2}}^{ \pm}\right|$are both constant.

Proposition 2.2. For a fixed $v \in T^{1} \tilde{S}$, the Busemann functions $b_{v}^{ \pm}$are strictly convex (along geodesics in $\tilde{S}$ ), are $C^{\infty}$, and have the property that $\left\|\nabla b_{v}^{ \pm}(p)\right\|=1$, for every $p \in \tilde{S}$.

Idea of Proof. For a fixed $t$, the functions

$$
p \mapsto d(\gamma(t), p)-t \quad \text { and } \quad d(\gamma(-t), p)-t
$$

are $C^{\infty}$, strictly convex and have gradient of constant norm 1 (this follows from Proposition 1.2). These properties hold uniformly in $t$. One shows using the exponential convergence in Proposition 1.2 that the $r$-jets of these 
functions converge uniformly on compact sets as $t \rightarrow \infty$, and so these properties carry to the limiting Busemann functions. $\diamond$

The nature of the dependence of $b_{v}^{ \pm}$on $v$ is a delicate matter, as the functions are are defined by a limiting process. In the current context of negatively curved surfaces, the functions $b^{ \pm}$are $C^{1}$; more precisely, the dependence of $b_{v}^{ \pm}(p)$ on $p \in \tilde{S}$ is $C^{\infty}$ (as explained in Proposition 2.2) and the dependence on $v \in T^{1} \tilde{S}$ is $C^{1}$. While the $C^{\infty}$ dependence of $b_{v}^{ \pm}(p)$ on $p$ holds for higher dimensional negatively curved manifolds, the $C^{1}$ dependence on $v$ is not a trivial fact and the analogous statement fails for general higher dimensional negatively curved manifolds (though Hölder continuity holds in general).

Horospheres (also called horocycles) are smooth submanifolds of $\tilde{S}$ defined as level sets of Busemann functions. For $v \in T^{1} \tilde{S}$, we define the (level 0) positive and negative horospheres of $v$ by

$$
H^{+}(v)=\left(b_{v}^{+}\right)^{-1}(0) \quad \text { and } H^{-}(v)=\left(b_{v}^{-}\right)^{-1}(0) .
$$

Proposition 2.2 implies that the horosphere $H^{+}(v)$ bounds a strictly geodesically convex region $B^{+}(v)=\left(b_{v}^{+}\right)^{-1}(0, \infty)$ called a horoball (similarly $H^{-}(v)$ bounds the convex horoball $\left.B^{-}(v)=\left(b_{v}^{+}\right)^{-1}(-\infty, 0)\right)$ The horospheres $H^{+}(v)$ and $H^{-}(v)$ are tangent at the basepoint of $v$ in $\tilde{S}$.

The terminology "horosphere" meaning roughly, "boundary sphere" is explained by the fact that horospheres are limits of geodesic spheres in $\tilde{S}$ as their centers tend to infinity along a fixed geodesic:

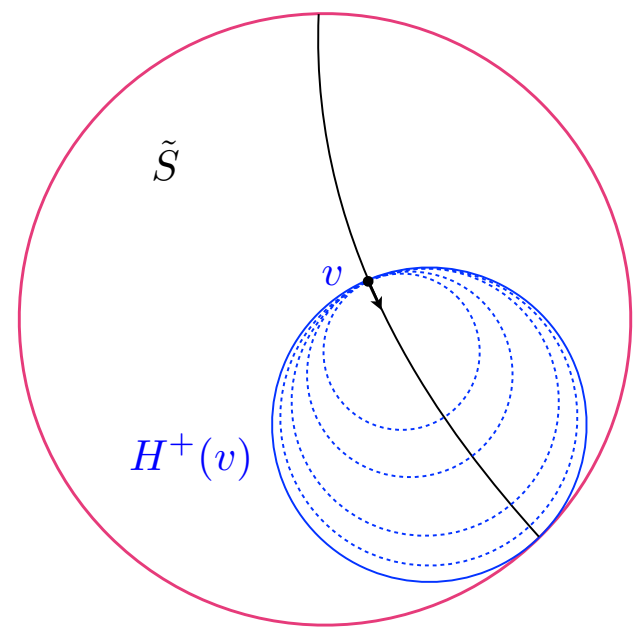

Figure 4. A horosphere is the limit of geodesic spheres.

To understand this, recall that the the Busemann function $b_{v}^{+}$is the limit: $b_{v}^{+}=\lim _{t \rightarrow \infty} b_{v, t}^{+}$, where $b_{v, t}^{+}(p)=d\left(\gamma_{v}(t), p\right)-t$; the level sets of the functions $b_{v, t}^{+}(p)$ are geodesic spheres, and the level set of $b_{v}^{+}$is the horosphere $H^{+}(v)$. 


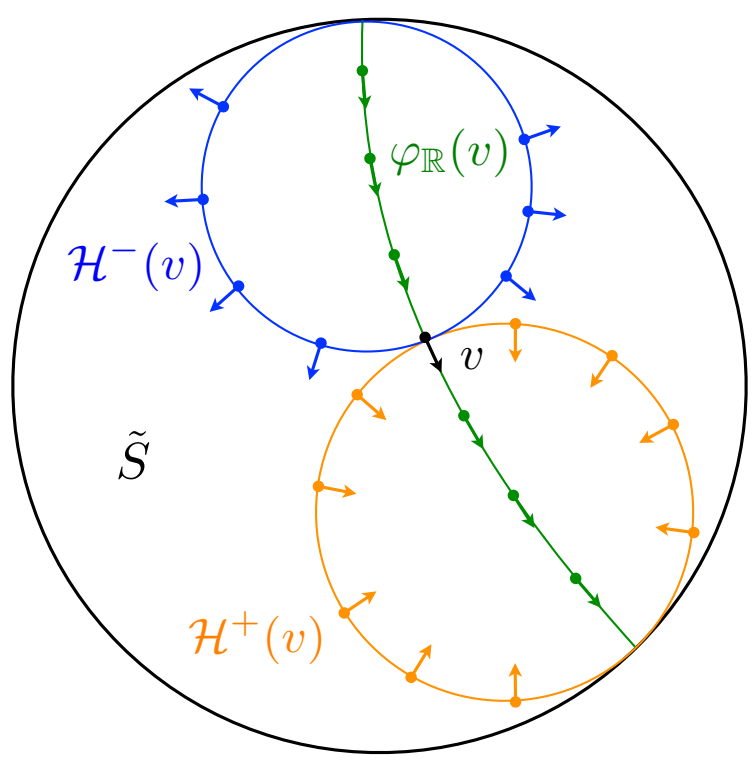

FiguRE 5. The geodesic flow orbit and horospheres through a vector $v \in T^{1} \widetilde{S}$.

Exercise 2.3. Show that on the hyperbolic plane horospheres are orbits of the upper and lower horocyclic (parabolic) subgroups:

$$
P^{+}=\left\{h_{t}^{+}:=\left(\begin{array}{cc}
1 & t \\
0 & 1
\end{array}\right): t \in \mathbb{R}\right\}
$$

and

$$
P^{-}=\left\{h_{t}^{-}:=\left(\begin{array}{ll}
1 & 0 \\
t & 1
\end{array}\right): t \in \mathbb{R}\right\} .
$$

In the disk model $\mathbb{D}$ of the hyperbolic plane, these horospheres are Euclidean circles tangent to the boundary $\partial \mathbb{D}$. The same is true in the upper half plane model $\mathbb{H}$, where the horizontal line $\operatorname{Im}(z)=$ const is regarded as a circle tangent to infinity.

The horospheres in $\tilde{S}$ lift naturally to horospheres in the unit tangent bundle $T^{1} \tilde{S}$, as follows. Proposition 2.2 implies that for a fixed $v \in T^{1} \tilde{S}$, the gradient vector fields $\nabla b_{v}^{ \pm}$are $C^{\infty}$ and have unit length. We define the (lifted) horosphere $\mathcal{H}^{ \pm}(v)$ in $T^{1} \tilde{S}$ through $v$ to be the restriction of $\nabla b_{v}^{ \pm}$to $H_{v}^{ \pm}$.

It follows directly from the definition of the Busemann functions (see Exercise 2.1) that:

$$
\varphi_{t}\left(\mathcal{H}^{ \pm}(v)\right)=\mathcal{H}^{ \pm}\left(\varphi_{t}(v)\right)
$$

and that any two positive (resp. negative) horospheres in $T^{1} \tilde{S}$ are either disjoint or coincide. They thus define a foliation of $T^{1} \tilde{S}$ called the positive 
(resp. negative) horospherical foliation. We denote these two foliations by $\mathcal{H}^{+}$and $\mathcal{H}^{-}$.

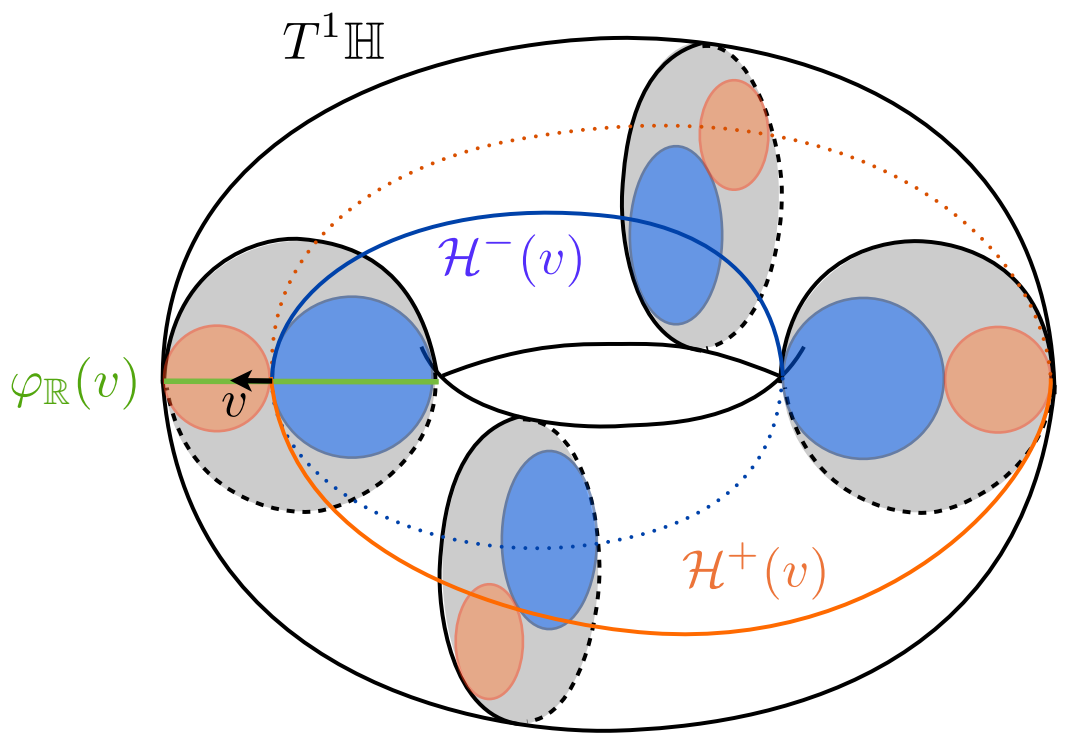

FiguRE 6. The geodesic and horospheres through a vector $v \in T^{1} \mathbb{H}$. The unit tangent bundle $T^{1} \mathbb{H}$ is a trivial circle bundle over the disk, depicted here as a solid torus.

Properties of these foliations are summarized in the following theorem.

Theorem 2.4. The horospherical foliations $\mathcal{H}^{+}$and $\mathcal{H}^{-}$have the following properties:

(1) Invariance under the geodesic flow: for each $v \in T^{1} \tilde{S}$ and $t \in \mathbb{R}:$

$$
\varphi_{t}\left(\mathcal{H}^{ \pm}(v)\right)=\mathcal{H}^{ \pm}\left(\varphi_{t}(v)\right)
$$

(2) Contraction under the geodesic flow: for each $v \in T^{1} \tilde{S}$ and $w \in \mathcal{H}^{+}(v)$ :

$$
\lim _{t \rightarrow \infty} d\left(\varphi_{t}(v), \varphi_{t}(w)\right)=0,
$$

and for each $w \in \mathcal{H}^{-}(v)$ :

$$
\lim _{t \rightarrow \infty} d\left(\varphi_{-t}(v), \varphi_{-t}(w)\right)=0 ;
$$

both converge exponentially fast in $t$.

(3) Horospheres are lines: the leaves of $\mathcal{H}^{+}$and $\mathcal{H}^{-}$are $C^{\infty}$ embedded submanifolds of $T^{1} \tilde{S}$, diffeomorphic to $\mathbb{R}$.

(4) Regularity of the foliations: The leaves of $\mathcal{H}^{ \pm}$(and their r-jets) depend in a $C^{1}$ fashion on their basepoints. 
(5) Transversality For each $v \in T^{1} \tilde{S}$, the angle between the tangent spaces $T_{v} \mathcal{H}^{+}(v)$ and $T_{v} \mathcal{H}^{-}(v)$ is bounded below away from 0 , uniformly over all $v \in T^{1} \tilde{S}$. Moreover, the vector $\dot{\varphi}(v)$ is transverse to the plane spanned by $T_{v} \mathcal{H}^{+}(v)$ and $T_{v} \mathcal{H}^{-}(v)$, uniformly.

Because the construction of Busemann functions is invariant under isometries of $\tilde{S}$, the horospherical foliations descend to foliations of $T^{1} S$ with analogous properties (though the leaves of these foliations are no longer embedded lines, but are instead densely immersed in $T^{1} S$ ).

There are two remaining relevant dynamical foliations of the unit tangent bundles, which we will call the weak horocyclic foliations $\mathcal{W}^{+}$and $\mathcal{W}^{-}$. The leaves of $\mathcal{W}^{ \pm}$are surfaces and are obtained from the leaves of $\mathcal{H}^{ \pm}$by applying the geodesic flow:

$$
\mathcal{W}^{ \pm}(v)=\varphi_{\mathbb{R}}\left(\mathcal{H}^{ \pm}(v)\right)
$$

In $T^{1} \tilde{S}$, the leaf of the weak foliation containing $v \in T^{1} \tilde{S}$ is just the image of the gradient vector field (which is a section of $T^{1} \tilde{S}$ over $\tilde{S}$ ):

$$
\mathcal{W}^{ \pm}(v)=\nabla b_{v}^{ \pm}(\tilde{S}) .
$$

Each leaf of $\mathcal{W}^{ \pm}$in $T^{1} \tilde{S}$ is thus naturally diffeomorphic to $\tilde{S}$ and is subfoliated both by orbits of the geodesic flow and by leaves of the corresponding horospherical foliation $\mathcal{H}^{ \pm}$.

The existence of the (exponentially contracted) positive and negative horocyclic foliations implies that the geodesic flow in negative curvature is an Anosov flow. Volume-preserving Anosov flows have strong dynamical properties. Some of them are summarized in the present context in the following theorem.

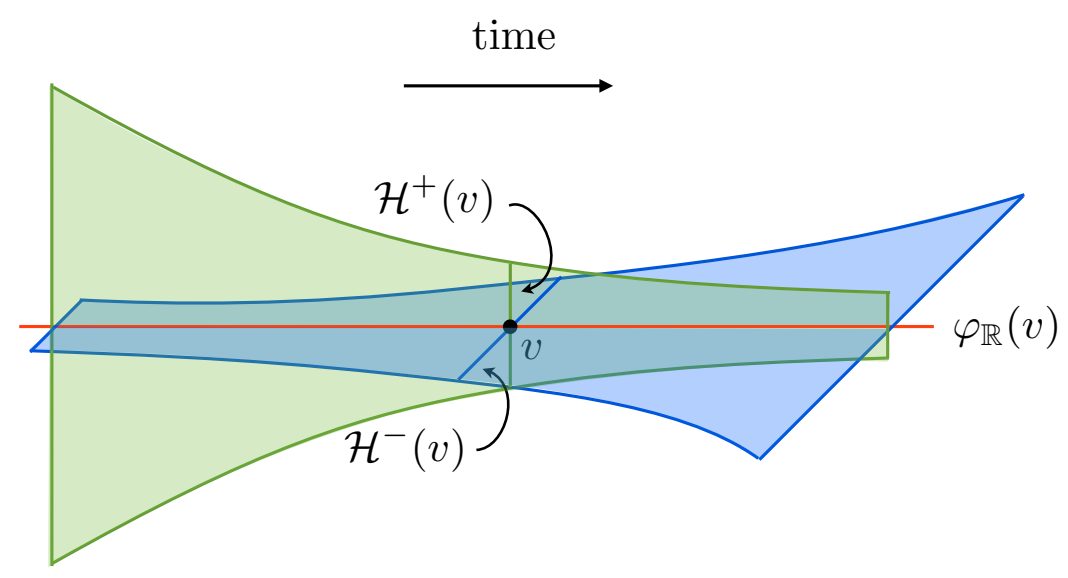

Figure 7. The Anosov property of the geodesic flow in negative curvature 
Theorem 2.5. Let $S$ be a closed, negatively curved surface, and let $\varphi_{t}: T^{1} S \rightarrow$ $T^{1} S$ be the geodesic flow. Then:

(1) the set of periodic orbits for $\varphi_{t}$ (which project to closed geodesics in $S$ ) is dense in $T^{1} S$ (Hedlund, 1930's),

(2) $\varphi_{t}$ is ergodic with respect to Liouville measure: if $A \subset T^{1} S$ is a Borel measurable set, and $\varphi_{t}(A)=A$ for all $t \in \mathbb{R}$, then either $\lambda(A)=0$ or $\lambda\left(T^{1} S \backslash A\right)=0$ (E. Hopf, 1930's),

(3) there exists $v \in T^{1} S$ whose orbit $\varphi_{\mathbb{R}}(v)$ is dense in $T^{1} S$ (this follows from (2); in fact almost every $v \in T^{1} S$ has a dense orbit).

Remark: By the Birkhoff Ergodic Theorem, ergodicity of $\varphi_{t}$ is equivalent to the following statement: for any integrable function $f: T^{1} S \rightarrow \mathbb{R}$, and for $\lambda$-a.e. $v \in T^{1} S$ :

$$
\lim _{T \rightarrow \infty} \frac{1}{T} \int_{0}^{T} f\left(\varphi_{t}(v)\right) d t=\frac{1}{\lambda\left(T^{1} S\right)} \int_{T^{1} S} f(v) d \lambda(v) .
$$

We will use this fact in the proof of Otal's theorem (although it is possible to use instead the weaker statement that $\varphi_{t}$ has a dense orbit).

For a more detailed discussion of Busemann functions in nonpositive curvature, see [4]. For a more general discussion of Busemann functions on CAT(0) spaces, see [6].

Exercise 2.6. Verify the assertions of Theorem 2.4 in the case of the hyperbolic plane. Recall from previous exercises that $T^{1} \mathbb{H}=\operatorname{PSL}(2, \mathbb{R})$, with the Sasaki/left-invariant metrics, and the geodesic flow acts by right multiplication by the one-parameter subgroup $A$.

Verify that the horocycle foliations $\mathcal{H}^{ \pm}$are the foliations by cosets of the horocyclic subgroups $P^{ \pm}$(fun fact: the leaves of these foliations are Sasaki geodesics in $\left.T^{1} \mathbb{H}\right)$. The commutators $\left[a_{t}, h_{s}^{ \pm}\right]$are relevant. If you prefer, work on the level of the Lie algebra.

2.2. The space of geodesics and the boundary at infinity. Most of Otal's proof is situated in the space of geodesics of the manifolds $S$ and $S^{\prime}$. The scheme of the proof is to construct a correspondence between these boundaries with certain additional properties that in the end force $S$ and $S^{\prime}$ to be isometric. We define the space of geodesics $\mathcal{G}=\mathcal{G}(S)$ of the surface $S$ :

$$
\mathcal{G}=\{[\gamma]: \gamma \text { is a unit speed geodesic in } \tilde{S}\} .
$$

There is a natural distance on $\mathcal{G}$, defined by:

$$
d\left(\left[\gamma_{1}\right],\left[\gamma_{2}\right]\right)=d_{T^{1} \tilde{S}}\left(\dot{\gamma}_{1}(\mathbb{R}), \dot{\gamma}_{2}(\mathbb{R})\right) .
$$

We next define $C^{\infty}$ charts on $\mathcal{G}$. Fix $w \in T^{1} \tilde{S}$, and let $\mathcal{G}_{w}$ be the set of oriented geodesic classes in $\mathcal{G}$ that intersect $\gamma_{w}$ transversely at an angle in $(0, \pi)$. For each class $[\gamma] \in \mathcal{G}_{w}$ let $x=x([\gamma])$ be the unique real number such that $\gamma_{w}(-x) \cap \gamma(\mathbb{R}) \neq \emptyset$, and let $\theta=\theta([\gamma]) \in(0, \pi)$ be the angle between 
the oriented curves $\gamma$ and $\gamma_{w}$ at this point of intersection. The pair $(x, \theta)$ defines global coordinates on $\mathcal{G}_{w}$ and gives an identification

$$
G_{w} \simeq \mathbb{R} \times(0, \pi) .
$$

Exercise 2.7. Prove that this defines a $C^{\infty}$ structure on $\mathcal{G}$ in which the distance is $C^{\infty}$. Can you guess the homeomorphism type of $\mathcal{G}$ ? (This will be answered in a later exercise).

Extend these charts to charts on $T^{1} \tilde{S}$ as follows. For a fixed vector $w \in$ $T^{1} \tilde{S}$, let $\hat{\mathcal{G}}_{w} \subset T^{1} \tilde{S}$ be the set of all $v \in T^{1} \tilde{S}$ such that $\left[\gamma_{v}\right] \in \mathcal{G}_{w}$. For each $v \in \hat{\mathcal{G}}_{w}$, let $y=y(v)$ be the unique time (moving backwards) where $\gamma_{v}$ intersects $\gamma_{w}$ :

$$
\gamma_{v}(-y)=\gamma(-x)=\gamma_{v}(\mathbb{R}) \cap \gamma_{w}(\mathbb{R}) .
$$

Show that $(x, \theta, y): \mathcal{G}_{w} \rightarrow \mathbb{R} \times(0, \pi) \times \mathbb{R}$ are $C^{\infty}$ coordinates on $T^{1} \tilde{S}$, and express the geodesic flow in these coordinates.

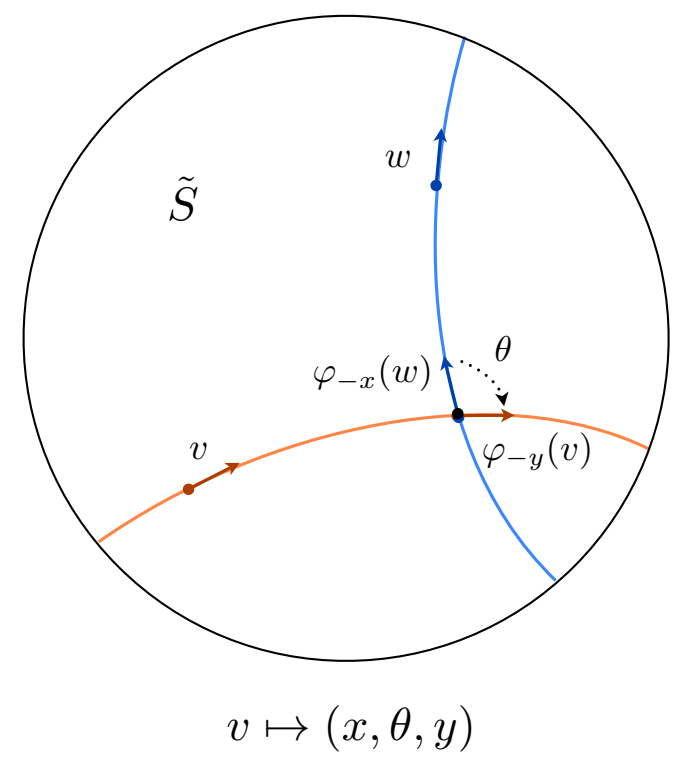

Figure 8. Coordinates on $T^{1} \tilde{S}$

Exercise 2.8. There is an involution sending $[\gamma(t)]$ to $[\gamma(-t)]$ (with the opposite orientation). Show that this action is free. The quotient $\mathcal{G} /[\gamma(t)] \sim$ $[\gamma(-t)]$ is the space of undirected geodesics. What is its homeomorphism type?

In the case of the hyperbolic plane, identify $\mathcal{G}$ with a coset space of $P S L(2, \mathbb{R})$. What is the involution?

Since $\pi_{1}(S)$ acts by isometries on $\tilde{S}$, it sends geodesics to geodesics. This induces a $\pi_{1}(S)$ action on $\mathcal{G}$, which is $C^{\infty}$. Unlike the action of $\pi_{1}(S)$ on $\tilde{S}$, 
this action is far from being discrete (though it is faithful). In fact, every orbit of this action is dense.

As we have just seen with the $C^{\infty}$ charts, any structure on $T^{1} \tilde{S}$ that is invariant under the geodesic flow gives rise to a corresponding structure on $\mathcal{G}$. If this structure is the pullback or lift of a structure on $T^{1} S$, then the corresponding structure on $\mathcal{G}$ is $\pi_{1}(S)$-invariant. An example of such a structure is each of the weak horospherical foliations of $T^{1} S$.

The weak horocyclic foliations $\mathcal{W}^{+}$and $\mathcal{W}^{-}$give rise to corresponding foliations $\mathcal{F}^{+}$and $\mathcal{F}^{-}$of $\mathcal{G}$. Fix a geodesic $[\gamma] \in \mathcal{G}$ and consider the set of all geodesics forward asymptotic to it (under some unit-speed parametrization). This defines the leaf $\mathcal{F}^{+}([\gamma])$ of a foliation $\mathcal{F}^{+}$, which is $C^{\infty}$, naturally diffeomorphic to $\mathcal{H}^{+}(\dot{\gamma}(0))$. Similarly, we define the leaf $\mathcal{F}^{-}([\gamma])$ of $\mathcal{F}^{-}$to be the set of geodesics asymptotic in backwards time to $[\gamma]$, which is $C^{\infty}$, naturally diffeomorphic to $\mathcal{H}^{+}(\dot{\gamma}(0))$. Since the foliations $\mathcal{W}^{ \pm}$are transversely $C^{1}$, so are the foliations $\mathcal{F}^{ \pm}$.

We next define the boundary $\mathcal{B}$ at infinity of $\tilde{S}$. From the point of view of a smooth dynamicist, this construction is quite different than the construction of the space of geodesics, as it involves choices that degrade the quality of the smooth structure at infinity.

We define the boundary $\mathcal{B}$ of $\tilde{S}$ to be the leaf space for $\mathcal{F}^{+}$:

$$
\mathcal{B}:=\mathcal{G} / \mathcal{F}^{+} \text {. }
$$

Each point $\xi \in \mathcal{B}$ corresponds to a leaf of $\mathcal{F}^{+}$, which is itself an equivalence class of forward-asymptotic geodesic rays. Since $S$ is a surface, the boundary of $\tilde{S}$ has a $C^{1}$ structure, since the foliation $\mathcal{F}^{+}$is transversely $C^{1}$. Since the foliation $\mathcal{F}^{+}$is invariant under the $\pi_{1}(S)$ action, there is an induced action of $\pi_{1}(S)$ on $\mathcal{B}$.

Exercise 2.9. Show that $\mathcal{B}$ is $C^{1}$ diffeomorphic to $\mathcal{G} / \mathcal{F}^{-}$and that both are $C^{1}$ diffeomorphic to the circle.

Exercise 2.10. Prove the following proposition:

Proposition 2.11. The foliations $\mathcal{F}^{+}$and $\mathcal{F}^{-}$are transverse to each other, are transversely $C^{1}$, and have $C^{\infty}$ leaves. There is a $C^{1}$ diffeomorphism between $\mathcal{G}$ and

$$
\mathcal{B} \times \mathcal{B} \backslash \Delta,
$$

Where $\Delta=\{(\zeta, \zeta): \zeta \in \mathcal{B}\}$ is the diagonal. This diffeomorphism sends the "horizontal" foliation

$$
\{(\mathcal{B} \backslash\{\zeta\}) \times\{\zeta\}: \zeta \in \mathcal{B}\}
$$

of $\mathcal{B} \times \mathcal{B} \backslash \Delta$ to the foliation $\mathcal{F}^{+}$and the "vertical" foliation

$$
\{\{\zeta\} \times(\mathcal{B} \backslash\{\zeta\}): \zeta \in \mathcal{B}\}
$$

to the foliation $\mathcal{F}^{+}$. This diffeomorphism conjugates the diagonal action of $\pi_{1}(S)$ on $\mathcal{B} \times \mathcal{B} \backslash \Delta$ with the natural action of $\pi_{1}(S)$ on $\mathcal{G}$. 
Exercise 2.12. Express the boundary $\mathcal{B}$ of the hyperbolic plane $\mathbb{H}$ as a homogeneous space of $S L(2, \mathbb{R})$. Write explicity in algebraic terms the diffeomorphism between $\mathcal{B}$ and $\mathcal{G} / \mathcal{F}^{+}$, and between $\mathcal{B} \times \mathcal{B} \backslash \Delta$ and $\mathcal{G}$.

Henceforth, we identify $\mathcal{G}$ with $\mathcal{B} \times \mathcal{B} \backslash \Delta$. The involution $I$ on $T^{1} \tilde{S}$ gives rise to the flip transformation $F: \mathcal{G} \rightarrow \mathcal{G}$ which switches the two $\mathcal{B}$ factors. This is the same involution described in the earlier exercise, sending $[\gamma(t)]$ to $[\gamma(-t)]$

The next structure on $\mathcal{G}$ that we construct is the so-called Liouville current, which is a Radon measure (locally finite) invariant under the action of $\pi_{1}(S)$ and the flip $F .^{3}$

The idea behind the construction is to start with Liouville measure on $T^{1} \tilde{S}$, which is invariant under the geodesic flow, the action of $\pi_{1}(S)$, and the involution $I$, and to "project" it to a measure on $\mathcal{G}$ invariant under the action of $\pi_{1}(S)$ and the flip map $F$. To do this, we express the tangent bundle $T^{1} \tilde{S}$ in local coordinates. Recall from Exercise 2.7 that each vector $w \in T^{1} \tilde{S}$ defines $C^{\infty}$ coordinates on $\hat{\mathcal{G}}_{w} \subset T^{1} \tilde{S}$ :

$$
(x, \theta, y): \hat{\mathcal{G}}_{w} \rightarrow \mathbb{R} \times(0, \pi) \times \mathbb{R} .
$$

The real numbers $\mathbb{R}$ act freely on $\hat{\mathcal{G}}_{w}$ by the geodesic flow and on $\mathbb{R} \times(0, \pi) \times \mathbb{R}$ by translation in the third factor. The coordinates are equivariant with respect to these $C^{\infty}$ actions. Taking the quotient by the action gives the $C^{\infty}$ coordinates:

$$
(x, \theta): \mathcal{G}_{w} \subset \mathcal{G} \rightarrow \mathbb{R} \times(0, \pi) .
$$

We claim that Liouville measure on $\hat{\mathcal{G}}_{w}$ in these coordinates is

$$
d \lambda(x, \theta, y)=\frac{1}{2} \sin \theta d \theta d x d y .
$$

This formula was first discovered by Emile Cartan; a proof of this fact can be found in [28, Section 19.3].

Exercise 2.13. Prove that the expression on the right hand side of (3) defines a measure on $T^{1} \tilde{S}$; that is, show that it is preserved under change of coordinates. (To do this exercise, it helps to have a good understanding of the properties of the geodesic flow and/or Jacobi fields).

It is clear that the measure defined by the expression on the right hand side of (3) is invariant under the geodesic flow and flip invariant. It is also clearly absolutely continuous with respect to volume on $T^{1} \tilde{S}$, with positive density $\frac{1}{2} \sin \theta$, and is invariant under the derivative action of the isometry group of $\tilde{S}$. These properties characterize $\lambda$ as Liouville measure, as claimed.

\footnotetext{
${ }^{3}$ More generally, a geodesic current is any Radon measure (locally finite) on $\mathcal{G}$ that is invariant under the actions of $\pi_{1}(S)$ and $F$. There is a $1-1$ correspondence between geodesic currents and flip-invariant finite invariant measures on $T^{1} S$. Geodesic currents were introduced by Bonahan in the hyperbolic setting but also have applications in geometric group theory [5, 15]. For an introduction to geodesic currents in $\mathbb{H}$, see [26].
} 
One cannot project Liouville measure directly from $\hat{\mathcal{G}}_{w}$ onto $\mathcal{G}_{w}$, because the fibers of $\hat{\mathcal{G}}_{w} \rightarrow \mathcal{G}_{w}$ are unbounded and have infinite conditional measure. Instead, using the geodesic flow, we restrict to a region in $\hat{\mathcal{G}}_{w}$ with bounded fibers and then project onto $\mathcal{G}_{w}$, as follows.

Viewing the surface $\{y=0\}$ in $\hat{\mathcal{G}}_{w}$ as a transversal to the geodesic flow, the slab $\mathcal{S}_{w}=\left\{(x, \theta, y):(x, \theta) \in \mathcal{G}_{w}, 0 \leq y<1\right\}$ is a fundamental domain for the action of the time- 1 map $\varphi_{1}$ of the geodesic flow on $\hat{\mathcal{G}}_{w}$. The Liouville current is obtained by projecting the Liouville measure in this slab $\mathcal{S}_{w}$ onto $\hat{\mathcal{G}}_{w}$.

In the local coordinates $(x, \theta)$ on the neighborhood $G_{w}$ described above, the Liouville current is given by:

$$
d m(x, \theta)=\frac{1}{2} \sin \theta d \theta d x .
$$

It is clear from this construction that $m$ is locally finite and invariant under isometries of $S$. Moreover it is absolutely continuous with respect to any smooth area form on $\mathcal{G}$, with smooth, positive density.

Exercise 2.14. Verify that for any geodesic segment $I \subset \tilde{S}$, the $m$-measure of the set of geodesics $[\gamma]$ meeting $I$ is equal to the geodesic length $\ell(I)$.

Exercise 2.15. Find a formula for the Liouville current on the Poincaré disk, identifying $\mathcal{G}$ with $\{|z|=1\}^{2} \backslash \Delta$ in the obvious way.

Answer: Up to a normalizing constant,

$$
d m(\xi, \eta)=\frac{|d \xi||d \eta|}{|\xi-\eta|^{2}}
$$

We summarize the structures defined up to this point:

- the space of geodesics $\mathcal{G}$ equipped with a $C^{\infty}$ action of $\pi_{1}(S)$,

- transverse foliations $\mathcal{F}^{+}$and $\mathcal{F}^{-}$invariant under the $\pi_{1}(S)$ action,

- a flip map $F: \mathcal{G} \rightarrow \mathcal{G}$ switching the foliations $\mathcal{F}^{+}$and $\mathcal{F}^{-}$, and commuting with the $\pi_{1}(S)$ action,

- a boundary space $\mathcal{B}$ equipped with a $C^{1}$ structure and a $C^{1}$ action of $\pi_{1}(S)$.

- a $C^{1}$ diffeomorphism, $\pi_{1}(S)$-equivariant, between $\mathcal{G}$ and $\mathcal{B} \times \mathcal{B} \backslash \Delta$ sending horizontal and vertical foliations to $\mathcal{F}^{-}$and $\mathcal{F}^{+}$, respectively, and conjugating the flip map on $\mathcal{G}$ with the coordinate flip on $\mathcal{B} \times$ $\mathcal{B} \backslash \Delta$,

- a Radon measure $m$ on $\mathcal{G}$ called the Liouville current, invariant under the action of $\pi_{1}(S)$ and the flip $F$.

In the next subsection we mention one more (closely related) structure on $\mathcal{G}$ called the symplectic cross ratio.

Exercise 2.16. The dynamics of $\pi_{1}(S)$ on $\mathcal{G}$ is closely connected to the dynamics of the geodesic flow on $T^{1} S$. Prove as many of the following equivalences as you can: 
(1) The geodesic flow has a dense orbit in $T^{1} S \Longleftrightarrow$ the action of $\pi_{1}(S)$ has a dense orbit in $\mathcal{G}$.

(2) Periodic orbits of the geodesic flow are dense in $T^{1} S \Longleftrightarrow$ fixed points for elements of the action of $\pi_{1}(S)$ are dense in $\mathcal{G}$.

(3) The geodesic flow is ergodic with respect to volume $\Longleftrightarrow$ the action of $\pi_{1}(M)$ is ergodic with respect to the Liouville current ${ }^{4}$.

Note that in the hyperbolic setting, where $\pi_{1}(S)$ embeds as a discrete subgroup $\Gamma<\operatorname{PSL}(2, \mathbb{R})$, the geodesic flow corresponds to the right action of the diagonal subgroup $A$ on the homogeneous space $\Gamma \backslash \operatorname{PSL}(2, \mathbb{R})$ and the action of $\pi_{1}(S)$ in $\mathcal{G}$ corresponds to the left action of $\Gamma$ on $\operatorname{PSL}(2, \mathbb{R}) / A$.

Exercise 2.17. In the Poincaré disk $\mathbb{D}$, the classical projective cross ratio of $\xi_{1}, \xi_{2}, \eta_{1}, \eta_{2} \in \partial \mathbb{D}$ is given by the formula:

$$
\left[\xi_{1}, \xi_{2}, \eta_{1}, \eta_{2}\right]=\frac{\left(\xi_{1}-\eta_{1}\right)\left(\xi_{2}-\eta_{2}\right)}{\left(\xi_{1}-\eta_{2}\right)\left(\eta_{1}-\xi_{2}\right)}
$$

Prove that

$$
m\left(\left[\xi_{1}, \xi_{2}\right] \times\left[\eta_{1}, \eta_{2}\right]\right)=\left|\log \left[\xi_{1}, \xi_{2}, \eta_{1}, \eta_{2}\right]\right| .
$$

2.3. The Liouville current, the cross ratio and the canonical contact form. The following figure gives a dynamical description of the Liouville current:
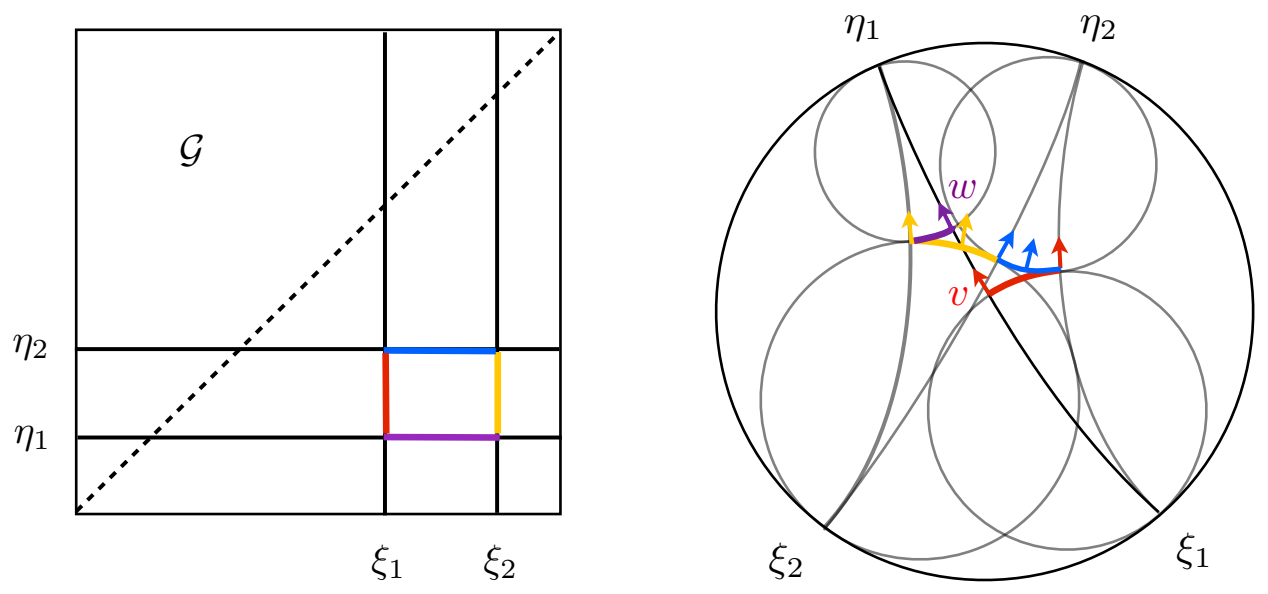

$$
\begin{gathered}
w=\varphi_{s}(v) \\
s=m\left(\left[\xi_{1}, \xi_{2}\right] \times\left[\eta_{1}, \eta_{2}\right]\right)
\end{gathered}
$$

FiguRE 9. The Liouville current expressed dynamically.

\footnotetext{
${ }^{4}$ meaning that if $A \subset \mathcal{G}$ is a Borel set satisfying $g(A)=A$, for all $g \in \pi_{1}(S)$, then either $m(A)=0$ or $m(\mathcal{G} \backslash A)=0$
} 
What this picture asserts is that the value of the Liouville current on a rectangle on $\mathcal{G}$ can be computed inside $T^{1} \tilde{S}$ : the recipe is as follows. Starting at any unit vector $v$ tangent the geodesic $\left(\xi_{1}, \eta_{1}\right)$, follow the leaf $\mathcal{H}^{-}(v)$ of the horocycle foliation through $v$ until you reach the unique unit vector $v^{\prime}$ on this leaf that is also tangent to the geodesic $\left(\xi_{2}, \eta_{1}\right)$. From this vector, follow the leaf $\mathcal{H}^{+}\left(v^{\prime}\right)$ of the opposite horocycle foliation until you reach the unique unit vector on this leaf that is also tangent to the geodesic $\left(\xi_{2}, \eta_{2}\right)$. Proceed in this fashion around the rectangle until you arrive back at a unit vector $w$ that is tangent to the geodesic $\left(\eta_{1}, \xi_{1}\right)$. The distance between $v$ and $w$ is the value of $m\left(\left[\xi_{1}, \xi_{2}\right] \times\left[\eta_{1}, \eta_{2}\right]\right)$. In particular, it does not depend on the choice of initial vector $v \in T^{1} S$.

Here is the same picture, but seen from inside the 3 -manifold $T^{1} \tilde{S}$ :

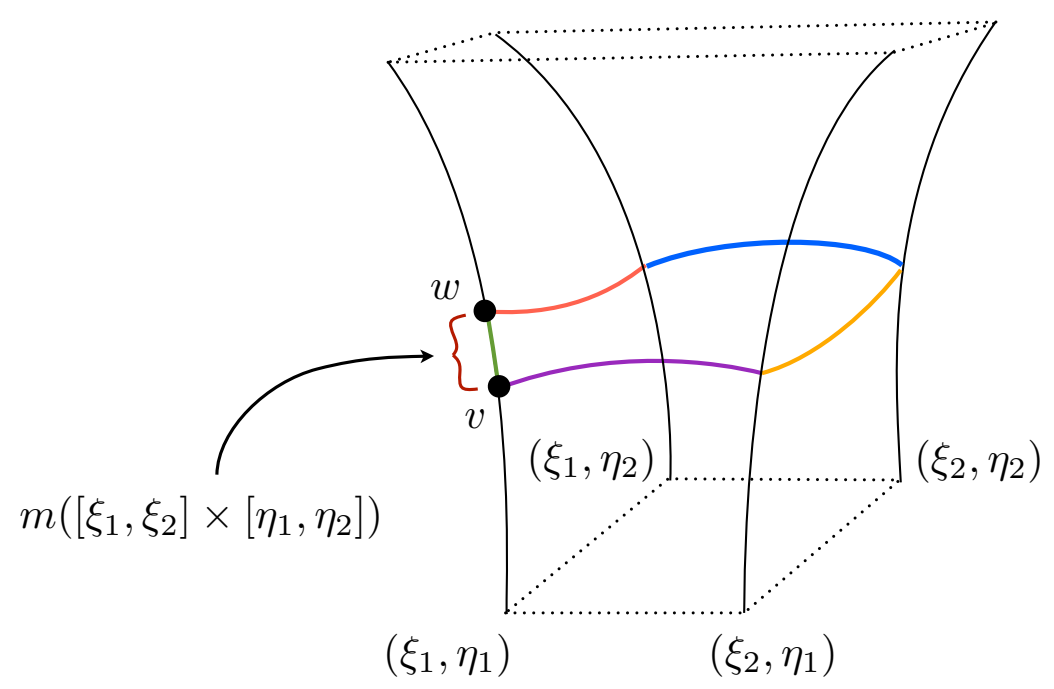

FiguRE 10. The Liouville current expressed dynamically inside of $T^{1} \tilde{S}$.

The reason this formula holds is Stokes's Theorem. Recall that the Liouville measure $\lambda$ is defined by the formula

$$
d \lambda=\alpha \wedge d \alpha,
$$

where $\alpha$ is the $\varphi_{t}$-invariant contact 1 -form on $T^{1} S$ described at the beginning of this section. This 1 -form $\alpha$ has the following properties:

(1) $\alpha(\dot{\varphi}) \equiv 1$, where $\dot{\varphi}$ is the vector field generating the geodesic flow on $T^{1} S$.

(2) If $\xi \in T\left(T^{1} S\right)$ is a vector tangent to either $\mathcal{H}^{+}$or $\mathcal{H}^{-}$, then $\alpha(\xi)=0$. The 2 -form $d \alpha$ is invariant under the geodesic flow. Since $d \lambda=\alpha \wedge d \alpha$, this 2 -form is nondegenerate on planes transverse to the flow. It plays the role of an area form on the space of geodesics, and the area it defines is nothing 
other than the Liouville current $m$. More precisely, if $\Pi: T^{1} \tilde{S} \rightarrow \mathcal{G}$ denotes the $C^{\infty}$ projection onto the space of geodesics, then $d \alpha=\Pi^{*} \beta$, where $\beta$ is a smooth 2-form giving the Liouville current $m$.

Thus to compute the Liouville current of a rectangle $\left[\xi_{1}, \xi_{2}\right] \times\left[\eta_{1}, \eta_{2}\right]$, we can use the formula:

$$
m\left(\left[\xi_{1}, \xi_{2}\right] \times\left[\eta_{1}, \eta_{2}\right]\right)=\left|\int_{\Sigma} d \alpha\right|
$$

where $\Sigma$ is any smooth surface in $T^{1} \tilde{S}$ that projects diffeomorphically (off of its boundary) under $\Pi$ onto $\left[\xi_{1}, \xi_{2}\right] \times\left[\eta_{1}, \eta_{2}\right]$ in $\mathcal{G}^{5}$

Now we can explain the formula in Figure 2.3. Let $\Sigma$ be a surface bounded by 5 arcs: two along leaves of $\mathcal{H}^{+}$, two along leaves of $\mathcal{H}^{-}$, and one along an orbit of $\varphi$, as shown in the following figure:

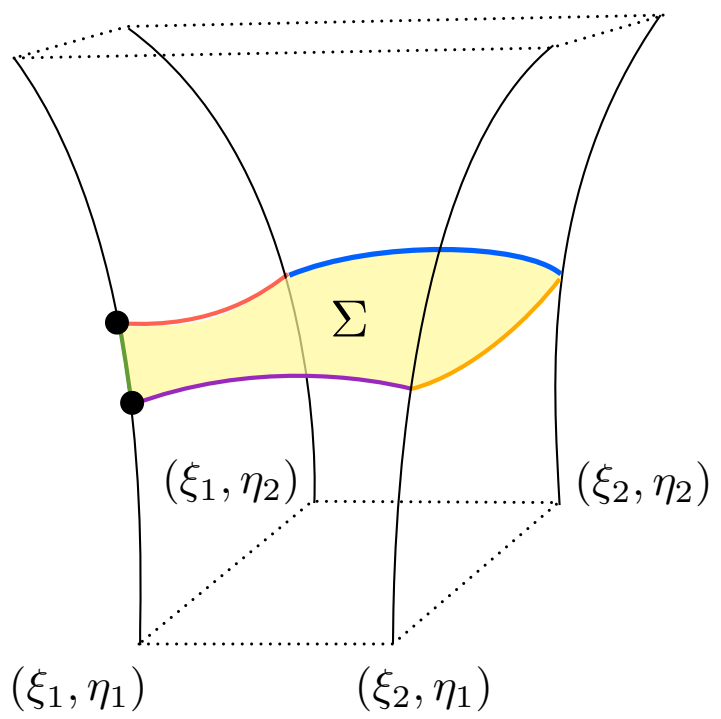

FiguRE 11 . The surface $\Sigma$.

Then Stokes's Theorem gives

$$
m\left(\left[\xi_{1}, \xi_{2}\right] \times\left[\eta_{1}, \eta_{2}\right]\right)=\left|\int_{\Sigma} d \alpha\right|=\left|\int_{\partial \Sigma} \alpha\right| .
$$

But $\alpha$ vanishes identically on vectors tangent to leaves of $\mathcal{H}^{ \pm}$and is identically 1 on the vector field $\dot{\varphi}$. Hence the integral of $\alpha$ over $\partial \Sigma$ is equal to the length of the one subarc of $\partial \Sigma$ that is contained in a $\varphi$ orbit. This is exactly what the formula in Figure 2.3 asserts.

A few words about the symplectic cross ratio. Since the Liouville current is a measure and not a differential form, it carries a little less information

\footnotetext{
${ }^{5}$ This can be taken as the definition of the Liouville current, from which the formula (4) then follows.
} 
than the 2 -form $d \alpha$. There is a structure defined on $\mathcal{G}$ that contains all of the information of $d \alpha$, called the symplectic cross ratio, which is a map

$$
[\cdot, \cdot, \cdot, \cdot]: \mathcal{B}^{4} \rightarrow \mathbb{R}_{>0}
$$

satisfying a collection of symmetry properties. In this context, one definition is:

$$
\left[\xi_{1}, \xi_{2}, \eta_{1}, \eta_{2}\right]=\exp \left(\int_{\Sigma} d \alpha\right)
$$

where $\Sigma$ is the surface described in Figure 2.3. Hence the cross-ratio and Liouville current are related by the formula:

$$
m\left(\left[\xi_{1}, \xi_{2}\right] \times\left[\eta_{1}, \eta_{2}\right]\right)=\left|\log \left[\xi_{1}, \xi_{2}, \eta_{1}, \eta_{2}\right]\right| .
$$

Notice that this generalizes the formula in Exercise 2.17 in the classical (hyperbolic) context. The short paper [23] has a nice discussion of the symplectic cross ratio.

It is also possible to define the cross ratio in much more general, geometric group-theoretic contexts, in the absence of a smooth structure. This type of definition proceeds via Busemann functions and is described, for example, in $[15]$.

The cross ratio makes an (uncredited) cameo appearance in Otal's proof, where he uses it to prove that the Liouville current is preserved under conjugacy (the analogue of Theorem 3.10 below). We discuss this more in Section 3.1.

2.4. Summary: a dictionary. To summarize, we have the following dictionary between the dynamics of the geodesic flow on $T^{1} S$ and the dynamics of the action of $\pi_{1}(S)$ on the space of geodesics $\mathcal{G}$ :

\begin{tabular}{|l|l|}
\hline Geodesic flow on $T^{1} S$ & Action of $\pi_{1}(S)$ on $\mathcal{G}$ \\
\hline weak invariant foliations $\mathcal{W}^{ \pm}$ & invariant foliations $\mathcal{F}^{ \pm}$ \\
time reversal $v \mapsto-v$ & flip map $(\xi, \eta) \mapsto(\eta, \xi)$ \\
Liouville measure $\lambda$ is invariant & Liouville current $m$ is invariant \\
transitivity ( $\exists$ a dense orbit) & transitivity $(\exists$ a dense orbit) \\
density of closed orbits & density of fixed points \\
ergodicity (with respect to $\lambda)$ & ergodicity (with respect to $m$ ) \\
invariant transverse 2-form $d \alpha$ & invariant symplectic cross-ratio \\
\hline
\end{tabular}

\section{LeCTURE 3}

We begin the proof of Otal's theorem. We start with:

Proposition 3.1. The space of negatively curved metrics on a closed surface $S$ is path connected. 
Idea of Proof. Hamilton's Ricci flow on surfaces preserves negative curvature and converges to a metric of constant negative curvature [19]. Given two metrics on a surface, flow both by Ricci flow to conformal metrics, and if necessary scale the metrics to have constant negative curvature -1 . Then both flowed metrics belong to Teichmüller space. Since Teichmüller space is connected, the two metrics can then be connected by a path through metrics of constant negative curvature. $\diamond$

Remark: The proof actually shows that Teichmüller space is a deformation retract of the space of negatively curved metrics on $S$. In high enough dimension, path-connectedness of the space of negatively curved metrics fails spectacularly. See [13]. On the other hand, it is possible to sidestep the issue of path-connectedness entirely by using the geometry of the universal cover of a negatively curved manifold (see Exercise 3.8 below).

Now let $S$ and $S^{\prime}$ denote negatively curved closed surfaces of the same genus. Later we will make the additional assumption that they have the same marked length spectra. We denote by $\varphi_{t}, \varphi_{t}^{\prime}$ their corresponding geodesic flows, $\mathcal{G}$ and $\mathcal{G}^{\prime}$ their corresponding spaces of geodesics, and so on.

Proposition 3.2. If the metrics on $S$ and $S^{\prime}$ (viewed as metrics on the same manifold $\Sigma_{g}$ ) are sufficiently $C^{2}$ close, then there exists an orbit equivalence between the geodesic flows $\varphi_{t}: T^{1} S \rightarrow T^{1} S$ and $\varphi_{t}^{\prime}: T_{1} S^{\prime} \rightarrow T^{1} S^{\prime}$; that is, there exists a homeomorphism $h: T^{1} S \rightarrow T^{1} S^{\prime}$ sending orbits of $\varphi_{t}$ to orbits of $\varphi_{t}^{\prime}$ :

$$
h\left(\varphi_{\mathbb{R}}(v)\right)=\varphi_{\mathbb{R}}^{\prime}(h(v)), \quad \forall v \in T^{1} S .
$$

This homeomorphism $h$ sends leaves of the weak horocyclic foliations $\mathcal{W}^{ \pm}$ to the corresponding leaves of $\mathcal{W}^{\prime \pm}$.

Remark: The existence of this orbit equivalence $h$ does not use the fact that $S$ and $S^{\prime}$ have the same marked length spectra, just the same genus.

Proof. The proof of Proposition 3.2 can follow more than one route. One is via the classical theory of Anosov flows, which we now discuss.

As explained in the previous lecture, the geodesic flows of $T^{1} S$ and $T^{1} S^{\prime}$ are normally hyperbolic (or "Anosov"). There is a very general stability theory for such dynamical systems (see [20]) which implies is particular that if the flows $\varphi_{t}$ and $\varphi_{t}^{\prime}$ are sufficiently $C^{1}$-close (which follows if the metrics are close in the $C^{2}$ topology), then there is a an orbit equivalence $h: T^{1} S \rightarrow T^{1} S^{\prime}$ (which is close to the identity in the $C^{0}$ sense). Moreover, this homeomorphism $h$ sends leaves of the weak horocyclic foliations $\mathcal{W}^{ \pm}$to the corresponding leaves of $\mathcal{W}^{ \pm \prime}$. $\diamond$

Such a homeomorphism $h$ is clearly not uniquely determined by these properties (for example $\varphi_{s^{\prime}}^{\prime} \circ h \circ \varphi_{s}$ for small, fixed $s, s^{\prime}$ will have the same properties), but the action of $h$ on orbits of $\varphi_{t}$ is unique. One can choose $h$ to be Hölder continuous and, in addition, $C^{\infty}$ along the orbits of $\varphi_{t}$ (see, e.g. 
[21]). For two general geodesic flows, this is the best one can get; in fact, we shall see that the orbit equivalence $h$ cannot be chosen to be a conjugacy of flows unless the surfaces are isometric.

Exercise 3.3. Prove that if $h$ is a conjugacy of flows, i.e.

$$
h \circ \varphi_{t}=\varphi_{t}^{\prime} \circ h, \quad \forall t \in \mathbb{R},
$$

then $h$ preserves the (strong) horocyclic foliations:

$$
h\left(\mathcal{H}^{ \pm}(v)\right)=\mathcal{H}^{ \pm \prime}(h(v)), \quad \forall v \in T^{1} S .
$$

Hint: The positive horocycle $\mathcal{H}^{+}(v)$ is the set of all $w \in T \tilde{S}$ such that

$$
\lim _{t \rightarrow \infty} d_{T^{1} \tilde{S}}\left(\varphi_{t}(v), \varphi_{t}(w)\right)=0 .
$$

Theorem 3.4. For any two negatively curved metrics on the genus $g$ surface $\Sigma_{g}$, the corresponding geodesic flows are orbit equivalent.

Proof. If the two metrics are sufficiently close, then this fact follows from the previous proposition. Path connectedness of the space of negatively curved metrics then implies the fact for any two negatively curved metrics. $\diamond$

We now upgrade the orbit equivalence given by Proposition 3.2 to an actual conjugacy of flows. This step uses the hypothesis that $S$ and $S^{\prime}$ have the same marked length spectrum. The following is an application of the so called Livsič theorem for transitive Anosov flows. It was proved independently by Guillemin and Kazhdan in their study of spectral rigidity.

Theorem 3.5. If $S$ and $S^{\prime}$ have the same marked length spectrum, then there exists a conjugacy $h: T^{1} \tilde{S} \rightarrow T^{1} \tilde{S}^{\prime}$ between the geodesic flows:

$$
h \circ \varphi_{t}=\varphi_{t}^{\prime} \circ h \text {. }
$$

Remark: The homeomorphism $h$ given by Theorem 3.5 is not unique, since for arbitrary $s, s^{\prime} \in \mathbb{R}$ the map $\varphi_{s^{\prime}} \circ h \circ \varphi_{s}$ also satisfies the conclusion. Up to such compositions, the conjugacy is unique.

Sketch of Proof. Fix an orbit equivalence $h_{0}$, which can be chosen to be Hölder continuous and $C^{\infty}$ along the orbits of $\varphi_{t}$ (this Hölder continuity condition is a technicality that is required to apply the Livsič theorem in the following).

Since $h_{0}$ is differentiable along the orbits of $\varphi$, there exists a Hölder continuous function $f: T^{1} S \rightarrow \mathbb{R}$ such that

$$
\left.\frac{d}{d t} h_{0}\left(\varphi_{t}(v)\right)\right|_{t=0}=f(v) \dot{\varphi}^{\prime}(h(v)),
$$

for all $v \in T^{1} S$. Let $a(v)=f(v)-1$.

The period of the orbit of $v \in T^{1} S$ under $\varphi_{t}$ is the infimum over $T>0$ satisfying $\varphi_{T}(v)=v$. If the period of $v$ is finite, then $v$ is said to be periodic and the orbit of $v$ is said to be closed or periodic. A closed orbit of a geodesic 
flow projects in the surface to closed geodesic, whose length is the period of the closed orbit.

Since $\ell_{S}=\ell_{S^{\prime}}$, and closed orbits of the geodesic flows $\varphi_{t}$ and $\varphi_{t}^{\prime}$ correspond to closed geodesics in $S$ and $S^{\prime}$, respectively, we have that the corresponding closed orbits of the two flows have the same periods.

This implies that for every $v \in T^{1} S$ of finite period $T>0$, we have

$$
\int_{0}^{T} a\left(\varphi_{t}(v)\right) d t=0
$$

A theorem of Livsič (see [21, Section 9.2]) implies there exists a function $A: M \rightarrow \mathbb{R}$, differentiable along orbits of $\varphi$ such that for every $v \in T^{1} S$ :

$$
a(v)=\left.\frac{d}{d t} A\left(\varphi_{t}(v)\right)\right|_{t=0}
$$

Let $h(v)=\psi_{-A(v)} \circ h_{0}(v)$. Then $h$ has the desired properties. $\diamond$

Remark: This argument generalizes to the following:

Theorem 3.6. Let $\varphi_{t}$ be a transitive (i.e. having a dense orbit) Anosov flow, and suppose that $\varphi_{t}$ is orbit equivalent to an Anosov flow $\varphi_{t}^{\prime}$ via a Holder homeomorphism that is $C^{1}$ along orbits. Suppose that corresponding closed orbits have the same period. Then the flows are conjugate via a homeomorphism (which is also Hölder continuous).

The homeomorphism given by Theorem 3.6 is not in general differentiable. It is differentiable in the setting of geodesic flows on negatively curved surfaces because further structure is preserved, namely the contact form $\alpha$.

Exercise 3.7. Prove that any homeomorphism $h: T^{1} S \rightarrow T^{1} S^{\prime}$ lifts to a homeomorphism $h: T^{1} \tilde{S} \rightarrow T^{1} \tilde{S}^{\prime}$. (Hint: the subgroup of $\pi_{1}\left(T^{1} S\right)$ generated by the fiber is the center of the group.) Prove that if $h$ is an orbit equivalence/conjugacy of the geodesic flows, then the lift $h: T^{1} \tilde{S} \rightarrow T^{1} \tilde{S}^{\prime}$ is a $\pi_{1}(S)$-equivariant orbit equivalence/conjugacy of the lifted geodesic flows.

Exercise 3.8. Denote the actions of $\Gamma=\pi_{1}(S)=\pi_{1}\left(S^{\prime}\right)$ on $\mathcal{G}, \mathcal{G}^{\prime}$ by $\rho, \rho^{\prime}$, where $\rho: \Gamma \rightarrow \operatorname{Diff}(\mathcal{G})$ and $\rho^{\prime}: \Gamma \rightarrow \operatorname{Diff}{ }^{\infty}\left(\mathcal{G}^{\prime}\right)$.

Prove the following:

Theorem 3.9. If $S$ and $S^{\prime}$ are homeomorphic, then there exists a unique homeomorphism $h: \mathcal{G} \rightarrow \mathcal{G}^{\prime}$ such that for every $g \in \Gamma$ :

$$
h \circ \rho(g)=\rho^{\prime}(g) \circ h \text {. }
$$

The map $h$ sends $\mathcal{F}^{ \pm}$to $\mathcal{F}^{ \pm^{\prime}}$ and satisfies $h \circ F=F^{\prime} \circ h$.

Hint: You can either deduce this from Theorem 3.4 or you can prove it directly using the following fact about negative curvature: Every quasigeodesic in $T^{1} \tilde{S}$ is a bounded distance from a unique geodesic. Proposition 1.2 can be used to prove continuity. This argument works in all dimensions. If two compact, negatively curved manifolds are homeomorphic, then their geodesic flows are orbit equivalent, in the sense of Theorem 3.4. 
The next result is the crux of the matter.

Theorem 3.10. The conjugacy $h$ sends the Liouville measure $\lambda$ on $T^{1} S$ to the Liouville measure $\lambda^{\prime}$ on $T^{1} S^{\prime}$. Moreover, the induced leaf conjugacy sends the Liouville current $m$ on $\mathcal{G}$ to the Liouville current $m^{\prime}$ on $\mathcal{G}^{\prime}$.

Remark: It is a general theme in low dimensional smooth dynamics that if two smooth, volume-preserving hyperbolic systems are conjugate via a homeomorphism preserving volume (or even just the volume class), then the conjugacy is in fact smooth. The simplest illustration of this principle is for expanding maps of the circle [29], and it is also true for Anosov diffeomorphisms of the 2-torus [12]. The same is true in this setting, and more: the conjugacy is not only smooth, but it induces an isometry.

Sketch of Proof. Recall that the Liouville current is the "projection" of Liouville measure onto the space $\mathcal{G}$; more precisely, in local coordinates in $\hat{\mathcal{G}}_{w}$ described above:

$$
d \lambda(x, \theta, y)=\frac{1}{2} \sin \theta d x d \theta d y=d m(\theta, x) d y,
$$

and similarly $d \lambda^{\prime}\left(x^{\prime}, \theta^{\prime}, y^{\prime}\right)=d m^{\prime}\left(\theta^{\prime}, x^{\prime}\right) d y^{\prime}$ in the coordinates on $\hat{\mathcal{G}}_{h(w)}^{\prime}$. Since $h$ conjugates the geodesic flow $\varphi_{t}$ to the flow $\varphi_{t}^{\prime}$, in these coordinates, $h$ must take the form:

$$
h(x, \theta, y)=\left(x^{\prime}(x, \theta, y), \theta^{\prime}(x, \theta, y), y^{\prime}+\alpha(x, \theta)\right)
$$

(verify this). It follows that $h$ sends $d \lambda$ to $h_{*}(d m) d y^{\prime}$. To show that $h_{*} \lambda=\lambda^{\prime}$ it thus suffices to show that $h_{*}(m)=m^{\prime}$.

We do this by using the dynamical description of the Liouville current summarized in Figure 9.

Since this picture is dynamical, drawn using horocycles and a piece of orbit of the geodesic flow, this picture is preserved by the conjugacy $h$ : the conjugacy sends pieces of $\varphi_{t}$-orbit isometrically onto pieces of pieces of $\varphi_{t}^{\prime}$ orbit, and Exercise 3.3 shows that $h$ sends the arcs in $\mathcal{H}^{ \pm}$to $\operatorname{arcs}$ in $\mathcal{H}^{\prime \pm}$. This means that $h$ preserves the current $m . \diamond$

Remark: In its full generality for Anosov flows on 3-manifolds, Theorem 3.10 can be stated as follows.

Theorem 3.11 (Feldman-Ornstein [14]). Let $M$ be a 3-manifold, and let $\alpha_{1}, \alpha_{2}$ be contact 1 -forms on $M$. Let $\varphi_{t}$ and $\psi_{t}$ be $C^{2}$ Anosov flows on $M$ such that $\varphi_{t}^{*}\left(\alpha_{1}\right)=\alpha_{1}$ and $\psi_{t}^{*}\left(\alpha_{2}\right)=\alpha_{2}$. Suppose there exists a homeomorphism $h$ such that such that $h \circ \varphi_{t}=\psi_{t} \circ h$, for every $t \in \mathbb{R}$. Then $h$ is $C^{1}$ and $h^{*}\left(\alpha_{2}\right)=\alpha_{1}$. 
3.1. Otal's Proof. There is another way to prove a version of Theorem 3.10, one that is employed by Otal. In this approach one uses an alternate description of the Liouville current/cross ratio in terms of intersection pairings with closed geodesics.

The rough idea is as follows. Exercise 2.14 shows that the set of all geodesics crossing an geodesic segment $I$ has measure $\ell(I)$ with respect to the Liouville current. On the other hand, we can express the set of geodesics in a square $\left[\xi_{1}, \xi_{2}\right] \times\left[\eta_{1}, \eta_{2}\right] \subset \mathcal{G}$ in terms of sets of geodesics crossing certain geodesic segments in $\tilde{S}$. There is a clean way to see this description, which was explained to me by Curt McMullen.

In the Klein model of the hyperbolic plane, the set $\mathcal{G} / F$ of undirected geodesics can be represented as points in $\mathbb{R} P^{2} \backslash \mathbb{H}^{2}$, as follows:

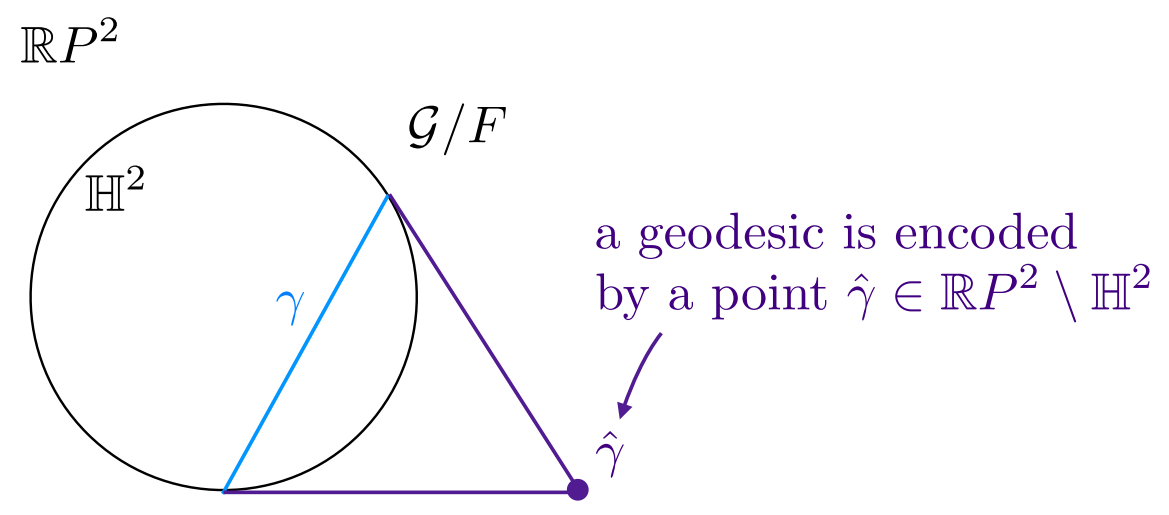

FiguRE $12 . \mathcal{G} / F \cong \mathbb{R} P^{2} \backslash \mathbb{H}^{2}$

In this model, the set of geodesics intersecting a fixed geodesic $\alpha$ is represented by a cone $\mathcal{C}_{\alpha}$ :

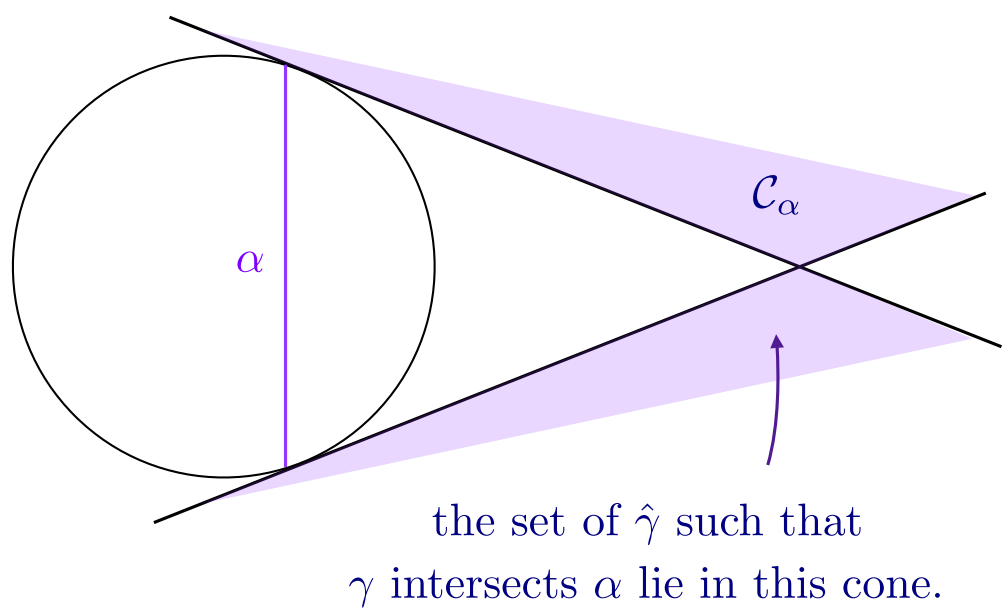


The set of geodesics connecting an interval $I \subset \mathcal{B}$ to an interval $J \subset \mathcal{B}$ is then a union of cones intersected with complements of cones:
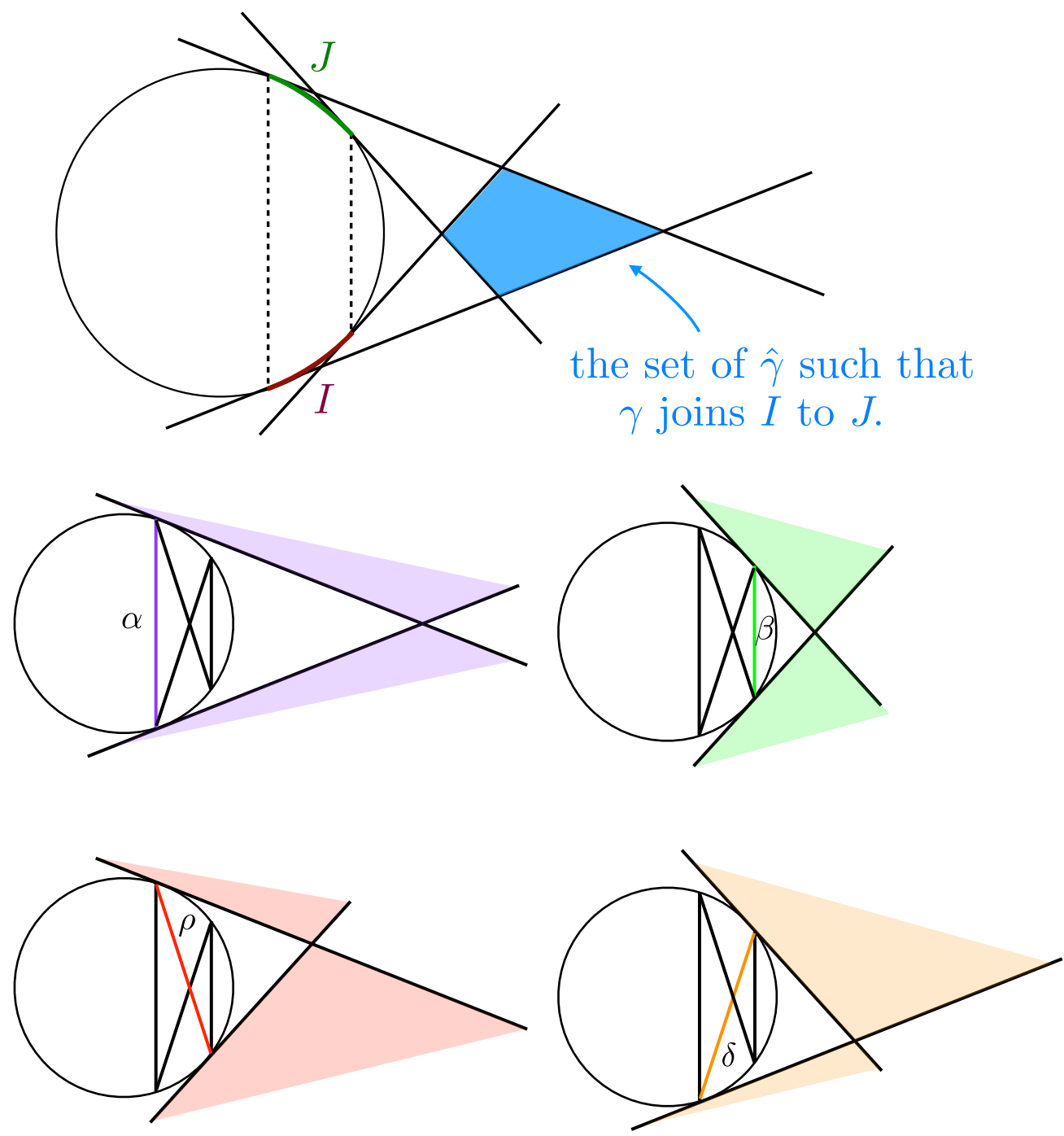

With respect to these labelings, we have

$$
I \times J=\mathcal{C}_{\delta} \cup \mathcal{C}_{\rho} \cap\left(\mathcal{G} \backslash \mathcal{C}_{\alpha}\right) \cap\left(\mathcal{G} \backslash \mathcal{C}_{\beta}\right),
$$

where points on the left are counted exactly twice in the right hand expression. Motivated by this idea, we attempt to define the Liouville current by

$$
m(I \times J)=\ell(\delta)+\ell(\rho)-\ell(\alpha)-\ell(\beta)
$$

(ignoring the factor of two that arises in the overcount). While the lengths of the geodesics $\alpha, \beta, \delta$ and $\rho$ are of course infinite, it turns out that this expression makes sense as a limit, when defined carefully. In particular, if 
we choose any 4 horospheres centered at $\xi_{1}, \xi_{2}, \eta_{1}, \eta_{2}$, as in the following figure, then the Liouville current can be computed this way:

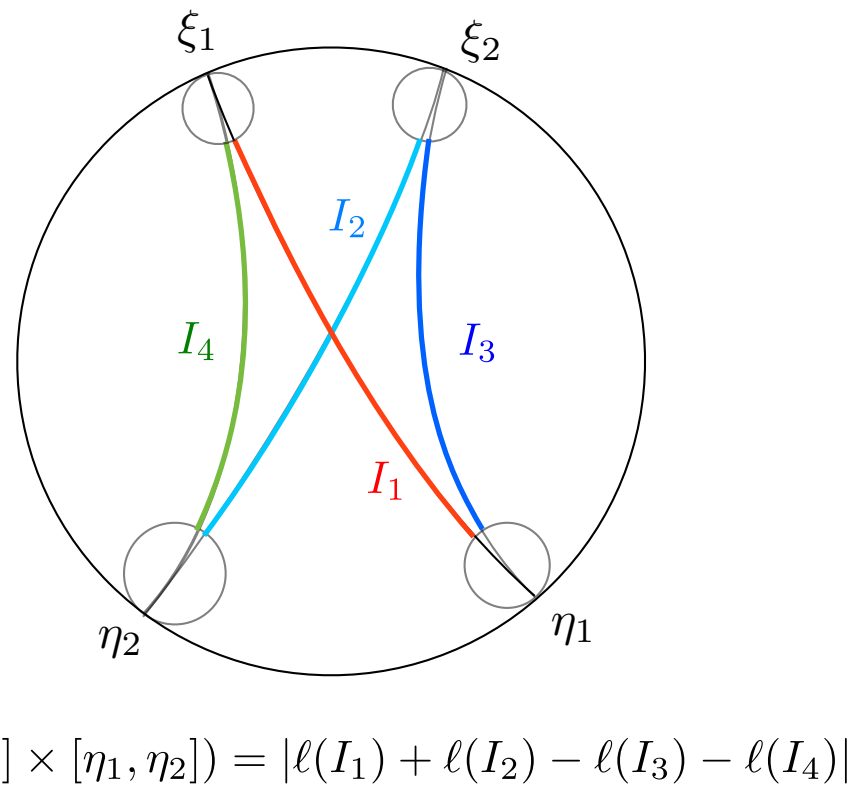

FiguRE 13. Otal's formula for the Liouville current.

This formula does not depend on the choice of horospheres, though one has to regard the lengths as signed distances in case the horospheres overlap. The systematic way to write this is using Busemann functions, which we leave as an exercise (see also [24, 15]).

Because this calculation does not depend on the choice of horospheres, we can see easily the connection between Otal's formula and the one given in Figure 9; Otal's formula is simply a generalization of the earlier one:
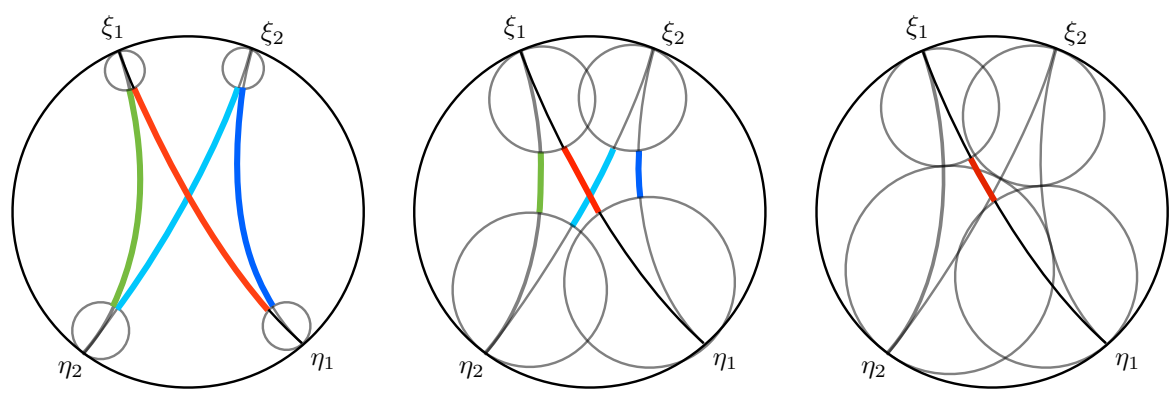

FiguRE 14. Otal's formula for the Liouville current: different choices of horospheres give the same answer. 
The proof of Otal's formula for the Liouville current is the same as the proof of the formula in Figure 9: one just chooses the correct surface $\Sigma$ in $T^{1} \tilde{S}$ projecting onto $\left[\xi_{1}, \xi_{2}\right] \times\left[\eta_{1}, \eta_{2}\right]$ and applies Stokes's Theorem. In fact, Otal proves that an analogous formula holds for any geodesic current, where the lengths " $\ell$ " are replaced by a suitable intersection pairing between currents and geodesics.

Exercise 3.12. Prove Otal's formula for the Liouville current.

In contrast with the proof presented here, Otal does not show explicitly in his proof that the geodesic flows for $S$ and $S^{\prime}$ are conjugate. Instead, he proves that the conjugacy on the space of geodesics sends the Liouville current $m$ to the current $m^{\prime}$. To do this, he shows that the current $m$ is determined by the lengths of the closed geodesics in $S$. He does this by choosing the points $\xi_{1}, \xi_{2}, \eta_{1}, \eta_{2}$ in Figure 13 so that the geodesics $\left(\eta_{1}, \xi_{1}\right)$ and $\left(\eta_{2}, \xi_{1}\right)$ are lifts of a single dense geodesic in $S$ (such a geodesic exists since the geodesic flow is transitive). Since such pairs are dense, the value of $m$ on such rectangles $\left[\xi_{1}, \xi_{2}\right] \times\left[\eta_{1}, \eta_{2}\right]$ determines the current $m$. Now by using the density of closed orbits for the geodesic flow (Theorem 2.5), he approximates the lengths of $I_{1}, I_{2}, I_{3}, I_{4}$ in Figure 13 arbitrarily well (by letting the horospheres tend to infinity) by the lengths of closed geodesics. Hence the lengths of closed geodesics determine the Liouville current, and if two negatively curved surfaces have the same marked length spectrum, then they have the same currents.

The marked length spectrum of course plays a role in both proofs. In the proof given here, the Livsič theorem is used to show that two surfaces with the same marked length spectrum have conjugate geodesic flows. Since such a conjugacy preserves length along geodesics and preserves the strong horocyclic foliations, it must preserve the Liouville current. Otal instead proves directly that the current is determined by the marked length spectrum, and so two surfaces with the same marked length spectrum have the same Liouville current.

\section{LECTURE 4}

We finish the proof. We are given a conjugacy of geodesic flows $h: T^{1} S \rightarrow$ $T^{1} S^{\prime}$, which lifts to a $\pi_{1}(S)$-equivariant conjugacy $h: T^{1} \tilde{S} \rightarrow T^{1} \tilde{S}$. Working backwards, if $h$ were indeed induced by an isometry between $S$ and $S^{\prime}$, then any three geodesics meeting in a single point would be sent by $h$ to three geodesics meeting at a point. Conversely, if any three geodesics meeting in a single point in $\tilde{S}$ are sent by $h$ to geodesics meeting in a single point in $\tilde{S}^{\prime}$, then $h$ defines a point map, sending the first point of intersection to the second. Once this point map is defined it is not difficult to see that it is an isometry, since it will also send geodesics to geodesics, preserving arclength.

Our strategy is to measure the defect in this property with a homeomorphism of $[0, \pi]$ that we will ultimately show is the identity map. Unlike the

previous lecture, the arguments in this lecture follow closely those in Otal's 
paper, with some simplifications due to the fact that we have established the existence of a conjugacy between geodesic flows.

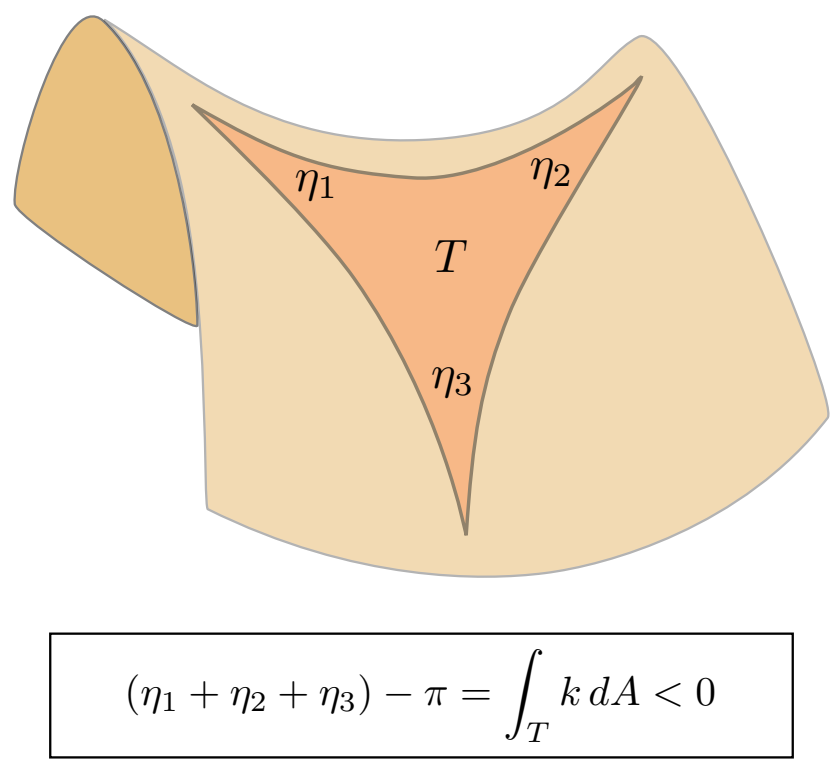

Figure 15. Gauss-Bonnet in negative curvature.

The intuition behind the proof is easy to explain. Consider three intersecting geodesics $\gamma_{1}, \gamma_{2}, \gamma_{3}$, forming angles $\theta_{1}, \theta_{2}, \theta_{3}$, with $\theta_{1}+\theta_{2}+\theta_{3}=\pi$. Under the conjugacy $h$, these geodesics are taken to geodesics $h\left(\gamma_{1}\right), h\left(\gamma_{2}\right), h\left(\gamma_{3}\right)$ forming a (possibly degenerate) geodesic triangle with angles $\eta_{1}, \eta_{2}, \eta_{3}$. The key observation is a consequence of the Gauss-Bonnet theorem:

Unless the area of this triangle is 0 , the sum of the angles $\eta_{1}+\eta_{2}+\eta_{3}$ is strictly less than $\pi$.

But if even one of these triangles is nondegenerate, then the map $h$ must be "dissipating angle," which violates the fact that $h$ preserves the Liouville volume.

To make this argument precise, we will introduce a function $F$ on the circle that measures the average dissipation of angle under $h$. For $\theta \in(0, \pi)$, and $v \in T^{1} \tilde{S}$, we denote by $\theta \cdot v$ the vector obtained from $v$ by rotating clockwise in the fiber of $T^{1} S$ through angle $\theta$.

Exercise 4.1. In $\operatorname{PSL}(2, \mathbb{R})$, find a 1-parameter subgroup $H \cong S^{1}$ such that this $S^{1}$-action on $T^{1} \mathbb{H} \cong \operatorname{PSL}(2, \mathbb{R})$ is given by right multiplication by $H$.

Define a function

$$
f: T^{1} \tilde{S} \times S^{1} \rightarrow S^{1}
$$

as follows. For $(v, \theta) \in T^{1} \tilde{S} \times S^{1}$, observe that the geodesics $\gamma_{v}$ and $\gamma_{\theta \cdot v}$ meet in a single point, at angle $\theta$. The images $h\left(\gamma_{v}\right)$ and $h\left(\gamma_{\theta \cdot v}\right)$ meet in 
a point as well; define $f(v, \theta)$ to be angle between them at their point of intersection.

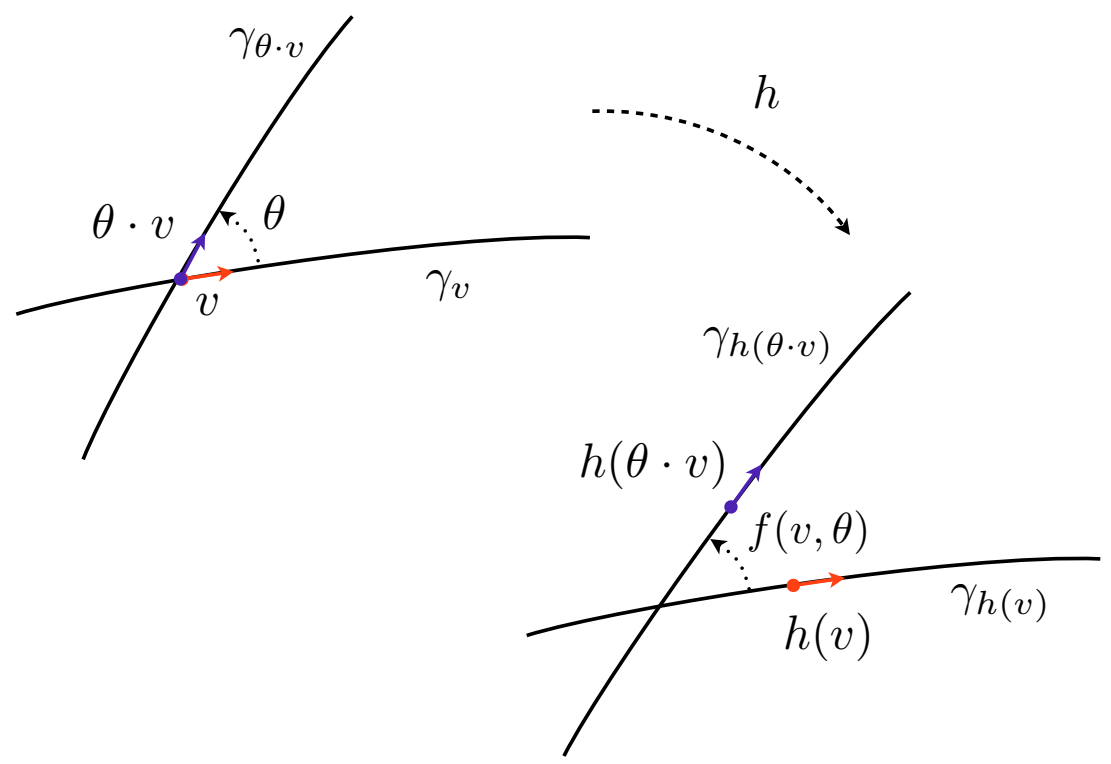

Figure 16. Definition of $f$.

Exercise 4.2. Prove that the conjugacy $h$ on the space of geodesics takes intersecting geodesics to intersecting geodesics.

Now define $F: S^{1} \rightarrow S^{1}$ by

$$
F(\theta)=\frac{1}{\lambda\left(T^{1} S\right)} \int_{T^{1} S} f(\theta, v) d \lambda(v),
$$

where $\lambda$ is the Liouville measure on $T^{1} S$.

Notice that the flip-invariance of $h$ on geodesics implies that $F(-\theta)=$ $-F(\theta)$. Let us focus on the restriction of $F$ to $[0, \pi]$. Two of its properties are summarized in the following proposition.

Proposition 4.3. [27, Proposition 6] The function $F:[0, \pi] \rightarrow[0, \pi]$ has the following properties:

(1) Symmetry about $\pi-\theta$ :

$$
F(\pi-\theta)=\pi-F(\theta)
$$

in particular, $F(\pi / 2)=\pi / 2$.

(2) Superadditivity: for all $\theta_{1}, \theta_{2}$, if $\theta_{1}+\theta_{2} \in[0, \pi]$, then

$$
F\left(\theta_{1}+\theta_{2}\right) \geq F\left(\theta_{1}\right)+F\left(\theta_{2}\right) .
$$




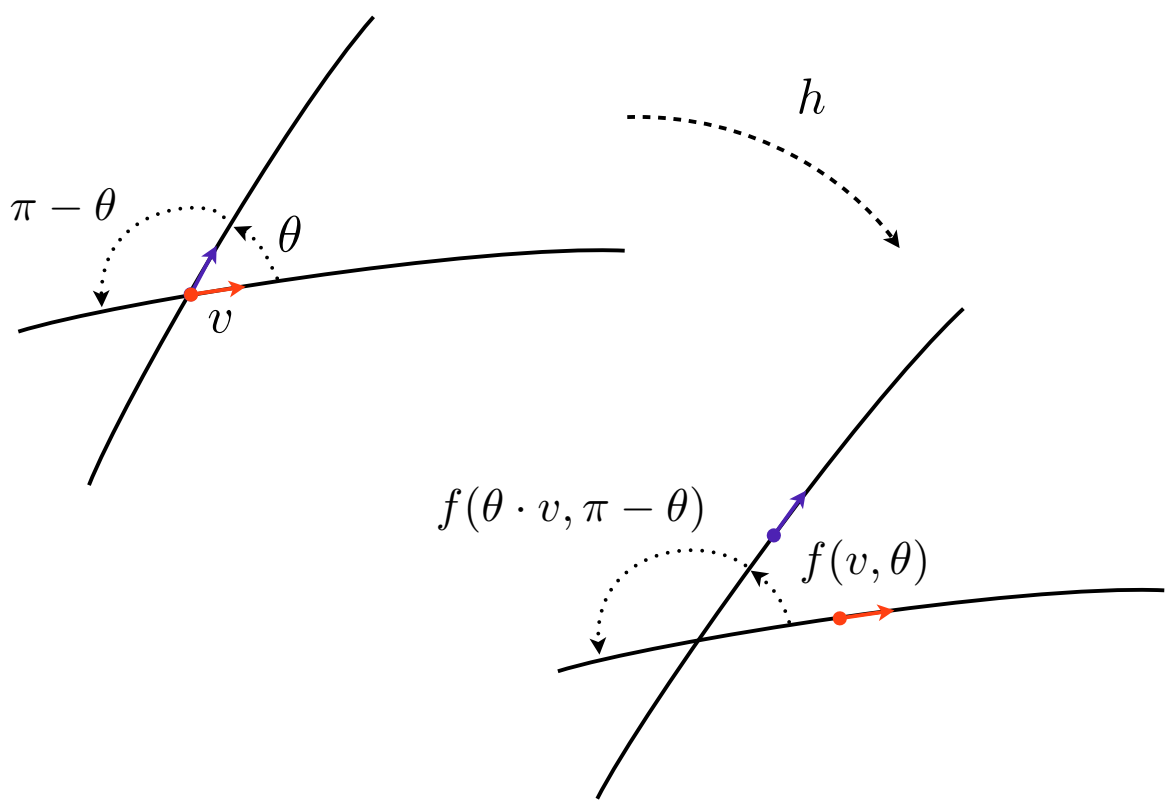

FiguRE 17. Proof of symmetry.

Proof. Part (1): We first show that:

$$
f(v, \theta)+f(\theta \cdot v, \pi-\theta)=\pi .
$$

This is easily seen by examining Figure 17. Integrate this expression with respect to $v$, using the fact that $\lambda$ is invariant under rotation in the fibers of $T^{1} S$, to get (1).

Part (2): We first establish that

$$
f\left(v, \theta_{1}\right)+f\left(\theta_{1} \cdot v, \theta_{2}\right) \leq f\left(v, \theta_{1}+\theta_{2}\right) .
$$

Let's recall the statement of the Gauss-Bonnet theorem for geodesic triangles. It is a direct consequence of Stokes's Theorem and the definition of curvature expressed graphically in Figure 1.1. It states that if $T$ is a region bounded by a geodesic triangle in a surface with Gaussian curvature $k$, then

$$
\int_{T} k d A=\left(\eta_{1}+\eta_{2}+\eta_{3}\right)-\pi,
$$

where $\eta_{1}, \eta_{2}, \eta_{2}$ are the interior angles of the triangle.

To see (5), observe from Figure 18 that the quantities $f\left(v, \theta_{1}\right), f\left(\theta_{1} \cdot v, \theta_{2}\right)$ and $\pi-f\left(v, \theta_{1}+\theta_{2}\right)$ measure the interior angles of a geodesic triangle in $\tilde{S}^{\prime}$.

Since $\tilde{S}^{\prime}$ has negative curvature, the Gauss-Bonnet Theorem then implies that the sum of these angles is less than or equal to $\pi$, with equality if and only if the triangle is degenerate. Integrate inequality (5) with respect to $v$, 


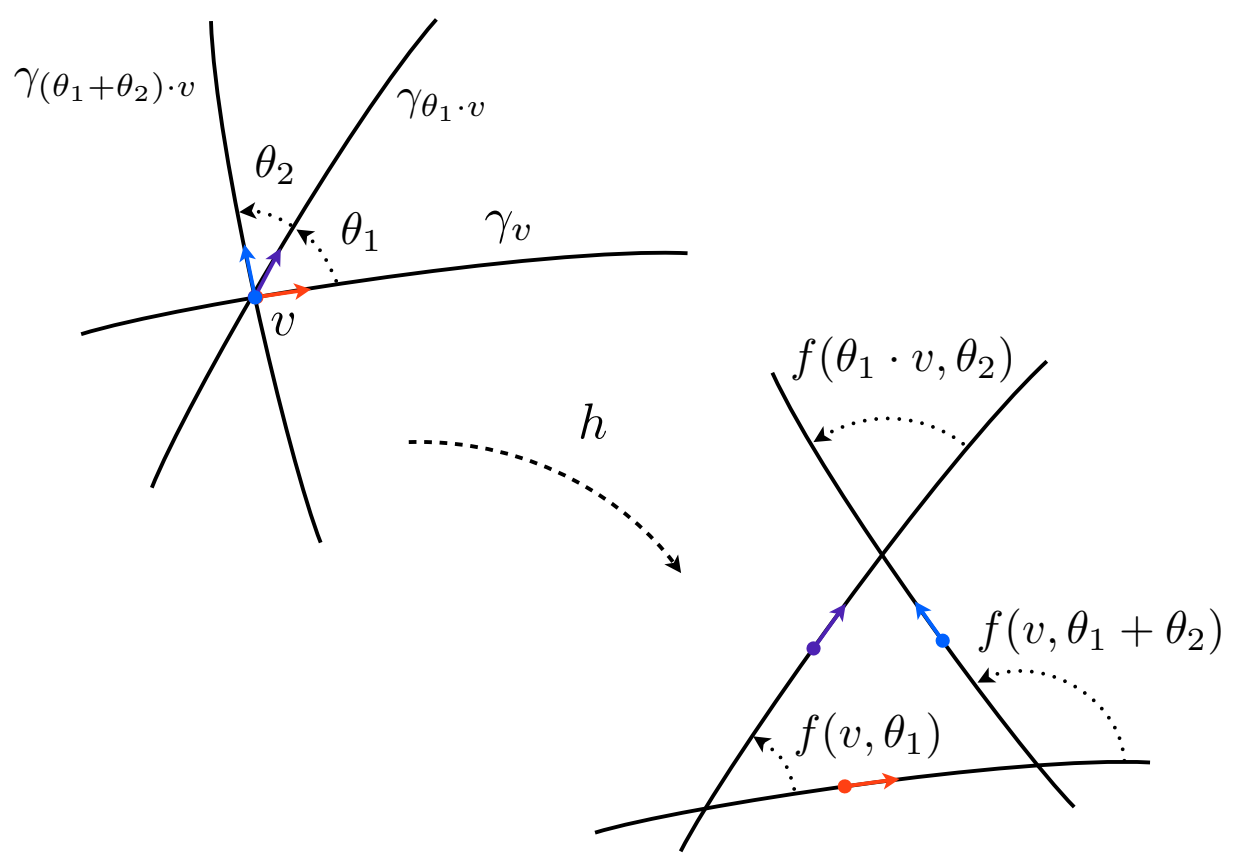

FIGURE 18. Proof of superadditivity.

using again the fact that $\lambda$ is invariant under rotation in the fibers, to get $(2) . \diamond$

Proposition 4.4. Let $G:[0, a] \rightarrow[0, a]$ be a superadditive function on the interval $[0, a]$ fixing the endpoints 0 and $a$. Then either:

(1) there exists $a_{0} \in(0, a]$ such that $G\left(a_{0}\right)=a_{0}$ and $G(x)<x$, for all $x \in\left(0, a_{0}\right)$, or

(2) $G(x) \leq x$ for all $x \in[0, a]$.

Proof. There are three cases to consider:

Case 1. There exists $a_{0} \in(0, a]$ such that $G\left(a_{0}\right)=a_{0}$, and $G(x)<x$, for all $x \in\left(0, a_{0}\right)$.

Case 2. There exists $a_{0} \in(0, a]$ such that $G\left(a_{0}\right)=a_{0}$, and $G(x)>x$, for all $x \in\left(0, a_{0}\right)$.

Case 3. There exists $a_{0} \in(0, a]$ such that $G(x)=x$, for all $x \in\left[0, a_{0}\right]$.

If Case 1 holds, then we are in conclusion (1) of the proposition. We claim that Case 2 cannot hold. Suppose that it does hold, and fix an $n>0$ such that $a / n<a_{0}$. For this $n$, superadditivity implies that $G(a) \geq n G(a / n)>$ $n a / n=a$, contradicting the fact that $G(a)=a$.

Suppose that Case 3 holds. We claim that $G(x) \leq x$, for all $x \in$ $[0, a]$. Consider first a point $x_{0} \in(0, a]$ satisfying $G\left(x_{0}\right)=x_{0}$. For $h \in$ 
$\left(0, \min \left\{x_{0}, a_{0}\right\}\right)$, superadditivity implies that $G\left(x_{0}-h\right) \leq G\left(x_{0}\right)-G(h)=$ $x_{0}-h$. Let $I \subset(0, a)$ be a maximal open interval such that $G(x)>x$ for all $x \in I$. If $I$ is not empty, then the right endpoint $x_{0}>0$ of $I$ is a fixed point for $G$ satisfying $G\left(x_{0}-h\right)>x_{0}-h$, for $h>0$ sufficiently small, which is a contradiction. $\diamond$

Proposition 4.5. (Compare $[27$, Proposition 7]) Let $F:[0, \pi] \rightarrow[0, \pi]$ be defined as above. Then for every $a \in[0, \pi]$ :

$$
\int_{0}^{a} \frac{\sin \theta}{\sin F(\theta)} d \theta \leq F(a)
$$

Proof. In the first step of the argument, we will use Jensen's Inequality. Recall that a function $J: \mathbb{R} \rightarrow \mathbb{R}$ is called convex if, for any two points $x, x^{\prime} \in \mathbb{R}$ and any $t \in[0,1]$,

$$
J\left(t x+(1-t) x^{\prime}\right) \leq t J(x)+(1-t) J\left(x^{\prime}\right) .
$$

Jensen's Inequality states that for any convex function $J$ and any probability measure $\mu$ on a space $X$, we have

$$
J\left(\int_{X} g(x) d \mu\right) \leq \int_{X} J(g(x)) d \mu(x),
$$

for every integrable real-valued function $g$ on $X$.

We would like to estimate $\int_{0}^{a} \sin \theta \csc F(\theta) d \theta$ from above; focusing on the second factor in the integrand, and applying Jensen's Inequality to the strictly convex function $\csc$ on $[0, \pi]$, we have

$$
\begin{aligned}
\csc F(\theta) & =\csc \left(\frac{1}{\lambda\left(T^{1} S\right)} \int_{T^{1} S} f(v, \theta) d \lambda(v)\right) \\
& \leq \frac{1}{\lambda\left(T^{1} S\right)} \int_{T^{1} S} \csc f(v, \theta) d \lambda(v) .
\end{aligned}
$$

Plugging this into the integral, we obtain

$$
\begin{aligned}
\int_{0}^{a} \frac{\sin \theta}{\sin F(\theta)} d \theta & \leq \int_{0}^{a}\left(\frac{1}{\lambda\left(T^{1} S\right)} \int_{T^{1} S} \csc f(v, \theta) d \lambda(v)\right) \sin \theta d \theta \\
& =\frac{1}{\lambda\left(T^{1} S\right)} \int_{T^{1} S}\left(\int_{0}^{a} \csc f(v, \theta) \sin \theta d \theta\right) d \lambda(v)
\end{aligned}
$$

by Fubini's Theorem.

We will now use the ergodicity of the flow $\varphi_{t}$ to rewrite this integral over $T^{1} S$ - a "space average" - as an integral over an orbit of the flow - a "time average."

A vector $v \in T^{1} S$ is said to be generic with respect to $\varphi_{t}$ and $\lambda$ if for every continuous function $k: T^{1} S \rightarrow \mathbb{R}$, we have

$$
\lim _{T \rightarrow \infty} \frac{1}{T} \int_{0}^{T} k\left(\varphi_{t}(v)\right) d t=\frac{1}{\lambda\left(T^{1} S\right)} \int_{T^{1} S} k(v) d \lambda(v) .
$$



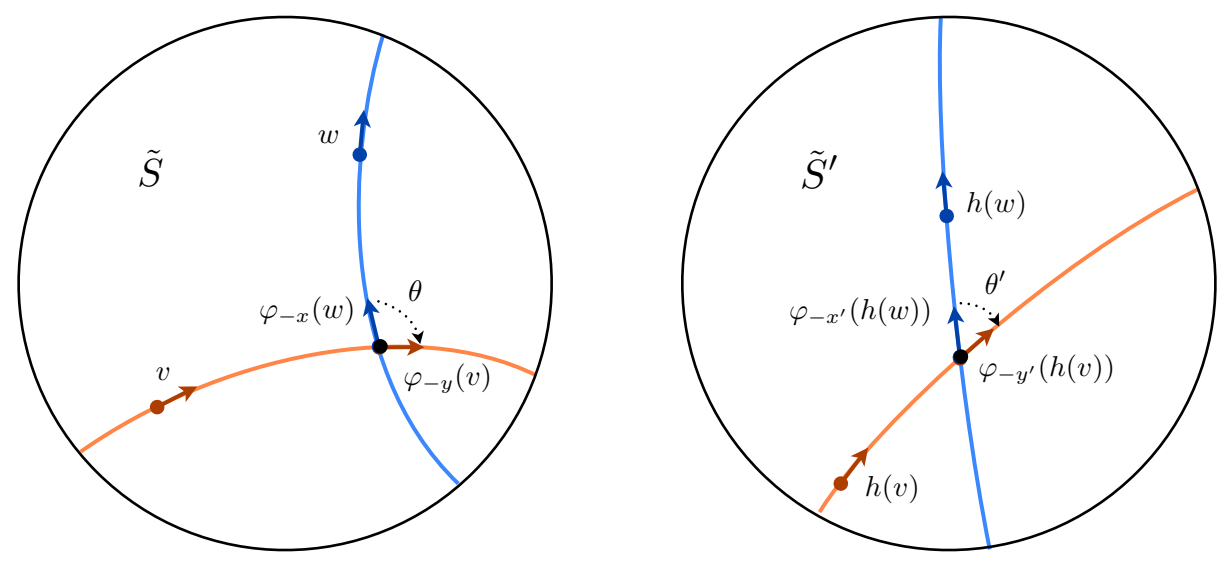

$\sin \theta d x d \theta d y \mapsto \sin \theta^{\prime} d x^{\prime} d \theta^{\prime} d y^{\prime}$

Figure 19. The effect of $h$ in coordinatees.

Ergodicity of $\varphi_{t}$ with respect to $\lambda$ implies that $\lambda$-almost every vector in $T^{1} S$ is generic.

Exercise 4.6. Prove this. The two main ingredients in the proof are the Birkhoff Ergodic Theorem and the fact that the continuous functions on $T^{1} S$ contain a countable dense subset.

Fix a generic $v_{0} \in T^{1} S$. Averaging the function

$$
k(v)=\int_{0}^{a} \csc f(v, \theta) \sin \theta d \theta
$$

along the orbit of $v_{0}$, we obtain:

$$
\begin{aligned}
\int_{0}^{a} \frac{\sin \theta}{\sin F(\theta)} d \theta & \leq \frac{1}{\lambda\left(T^{1} S\right)} \int_{T^{1} S}\left(\int_{0}^{a} \csc f(v, \theta) \sin \theta d \theta\right) d \lambda(v) \\
& =\lim _{T \rightarrow \infty} \frac{1}{T} \int_{0}^{T}\left(\int_{0}^{a} \csc f\left(\varphi_{t}\left(v_{0}\right), \theta\right) \sin \theta d \theta\right) d t
\end{aligned}
$$

The next step is to perform a change of coordinates, using the fact that the conjugacy $h$ preserves the Liouville current. In the $(x, \theta, y)$-coordinates based at $v_{0}$, the point $\theta \cdot \varphi_{t}\left(v_{0}\right)$ takes the form $(t, \theta, 0)$. Expressing $f$ with respect to the corresponding coordinates $\left(x^{\prime}, \theta^{\prime}, y^{\prime}\right)$ based at $h\left(v_{0}\right)$ in $T^{1} \tilde{S}^{\prime}$, we have

$$
f\left(\varphi_{t}\left(v_{0}\right), \theta\right)=\theta^{\prime}(h(t, \theta))
$$


Exercise 4.7. Show that the change of variables $(\theta, x) \mapsto\left(\theta^{\prime}, x^{\prime}\right)$ takes the region $[0, a] \times[0, T]$ to a region $R$ satisfying

$$
\begin{aligned}
& \left\{\left(\theta^{\prime}, x^{\prime}\right): 0 \leq \theta^{\prime} \leq f\left(\varphi_{x^{\prime}}\left(v_{0}\right), a\right) ; x^{\prime} \in[0, T-C]\right\} \subseteq \\
& R \subseteq\left\{\left(\theta^{\prime}, x^{\prime}\right): 0 \leq \theta^{\prime} \leq f\left(\varphi_{x^{\prime}}\left(v_{0}\right), a\right) ; x^{\prime} \in[0, T+C]\right\},
\end{aligned}
$$

for a constant $C>0$ that is independent of $T$.

Since $h$ preserves the Liouville current, we also have:

$$
h^{*}\left(\sin \theta^{\prime} d \theta^{\prime} d x^{\prime}\right)=\sin \theta d \theta d x .
$$

Thus the change of variables formula gives:

$$
\begin{aligned}
\frac{1}{T} \int_{0}^{T} \int_{0}^{a} \csc f\left(\varphi_{t}\left(v_{0}\right)\right. & , \theta) \sin \theta d \theta d t \\
& =\frac{1}{T} \int_{0}^{T} \int_{0}^{a} \csc \theta^{\prime}(h(x, \theta)) \sin \theta d \theta d x \\
& =\frac{1}{T} \iint_{h^{-1}(R)} \csc \theta^{\prime}(h(x, \theta)) h^{*}\left(\sin \theta^{\prime} d \theta^{\prime} d x^{\prime}\right) \\
& =\frac{1}{T} \iint_{R} \csc \theta^{\prime} \sin \theta^{\prime} d \theta^{\prime} d x^{\prime} \\
& =\frac{1}{T} \int_{0}^{T} \int_{0}^{f\left(\varphi_{x^{\prime}}\left(v_{0}\right), a\right)} \csc \theta^{\prime} \sin \theta^{\prime} d \theta^{\prime} d x^{\prime}+O\left(\frac{1}{T}\right) \\
& =\frac{1}{T} \int_{0}^{T}\left(\int_{0}^{f\left(\varphi_{x^{\prime}}\left(v_{0}\right), a\right)} d \theta^{\prime}\right) d x^{\prime}+O\left(\frac{1}{T}\right) \\
& =\frac{1}{T} \int_{0}^{T} f\left(\varphi_{t}\left(v_{0}\right), a\right) d t+O\left(\frac{1}{T}\right)
\end{aligned}
$$

Hence:

$$
\begin{aligned}
\int_{0}^{a} \frac{\sin \theta}{\sin F(\theta)} d \theta & \leq \lim _{T \rightarrow \infty} \frac{1}{T} \int_{0}^{T}\left(\int_{0}^{a} \csc f(v, \theta) \sin \theta d \theta\right) d t \\
& =\lim _{T \rightarrow \infty} \frac{1}{T} \int_{0}^{T} f\left(\varphi_{t}\left(v_{0}\right), a\right) d t
\end{aligned}
$$

The fact that $v_{0}$ is generic (this time applied to the function $k(v)=f(v, a)$ ), implies that this final limit exists, and furthermore:

$$
\begin{aligned}
\int_{0}^{a} \frac{\sin \theta}{\sin F(\theta)} d \theta & \leq \lim _{T \rightarrow \infty} \frac{1}{T} \int_{0}^{T} f\left(\varphi_{t}\left(v_{0}\right), a\right) d t \\
& =\frac{1}{\lambda\left(T^{1} S\right)} \int_{T^{1} S} f(v, \theta) d \lambda(v) \\
& =F(a) .
\end{aligned}
$$

$\diamond$ 
It is now a straightforward matter to complete the proof: we have illuminated a tension between the superadditivity of $F$, which by Proposition 4.4 implies a type of convexity of $F$ near 0 , and the fact that $h$ preserves volume, which by Proposition 4.5 implies a competing type of concavity near 0 . The only resolution of these competing properties is for $F$ to be the identity function. This will imply that $h$ takes triply intersecting geodesics to triply intersecting geodesics. As described above, we then have a map from $S$ to $S^{\prime}$ sending geodesics to geodesics. A little more work shows that this map is an isometry.

More precisely, Proposition 4.5 implies that there cannot be an interval $(0, a)$ on which $F(\theta)<\theta$ : otherwise, fix a maximal such interval, whose right endpoint $a>0$ is fixed by $F$. Since $F(\pi / 2)=\pi / 2$, we may assume that $a \leq \pi / 2$. Proposition 4.5 implies that

$$
\int_{0}^{a} \frac{\sin \theta}{\sin F(\theta)} d \theta \leq F(a)=a
$$

since $a \leq \pi / 2$ and $F(\theta)<\theta$ on $(0, a)$, the integrand on the left is strictly bounded below everywhere by 1 on $(0, a)$, and so the integral itself is bounded strictly below by $a$. But this gives the contradiction $a<a$.

Since there is no interval $(0, a)$ on which $F(a)<a$, it follows from Proposition 4.4 that $F(\theta) \leq \theta$ for all $\theta \in[0, \pi]$. Symmetry of $F$ with respect to $\pi-\theta$ implies that $F(\theta)=\theta$, and hence that $f(v, \theta)=\theta$. Going back to our earlier discussion, because the curvature of $S^{\prime}$ is strictly negative, this can occur only when $h$ sends every triple of intersecting geodesics to a triple of intersecting geodesics.

In $(x, \theta)$ coordinates based at some vector $v \in T^{1} S$ and the corresponding $\left(x^{\prime}, \theta^{\prime}\right)$ coordinates based at $h(v)$, we have that $\theta^{\prime}(h(x, \theta))=\theta$. Because the geodesics through vectors $h(\theta \cdot v)$ all meet at a single point, $x^{\prime}(h(x, \theta))$ does not depend on $\theta$; since $h^{*}\left(\sin \theta^{\prime} d x^{\prime} d \theta^{\prime}\right)=\sin \theta d x d \theta$, this can only happen if $x^{\prime}(h(x, \theta))=x+c$, where $c=c(v)$ is a constant that does not depend on $x$ or $\theta$. The function $v \mapsto c(v)$ defined on $T^{1} S$ is invariant under the geodesic flow $\varphi_{t}$; ergodicity implies that it is constant: $c(v)=c$, almost everywhere, and hence everywhere, since $c$ is continuous. Finally, let $\hat{h}(v)=\varphi_{c}^{\prime} \circ h$, where $c$ is the constant value of the function $c(v)$. We end the proof with an exercise:

Exercise 4.8. Prove that $\hat{h}$ sends fibers of $T^{1} \tilde{S}$ to fibers of $T^{1} \tilde{S}^{\prime}$ and projects to an isometry between $\tilde{S}$ and $\tilde{S}^{\prime}$.

\section{Final Comments}

The Otal-Croke result is just the beginning, of course. Similar results have been shown for other classes of manifolds, and even singular manifolds and orbifolds. One bothersome case which has not been fully understood is the case of negatively curved compact manifolds in higher dimension. What is easy to see is the following: if two negatively curved metrics on the same 
manifold have the same marked length spectrum, then their geodesic flows are conjugate. This boils down to the Livsič theory argument described in Lecture 3. What is not known is whether this conjugacy must be $C^{1}$ and volume-preserving (as is the case with surfaces) and more generally whether the conjugacy induces an isometry.

Some partial results have been established by Hamendstädt, using a powerful result of Besson-Courtois-Gallot $[18,2,3]$. The latter BCG result characterizes all negatively curved locally symmetric manifolds by two quantities: their volume and entropy. Among other things, their results imply that if a negatively curved metric on a locally symmetric compact manifold has the same volume and entropy as its locally symmetric counterpart, then the two Riemann structures are isometric. If the geodesic flows on these manifolds are conjugate, then their entropies coincide. Hamendstädt showed that in this context, their volumes must coincide as well; it follows that a compact, negatively curved manifold with the same marked length spectrum as a compact negatively curved locally symmetric manifold $M$ is in fact isometric to $M$.

In nonpositive curvature and beyond, there are many isospectral rigidity results: too many to mention here. The survey [9] contains a discussion of some of these. Many of these results and questions are phrased in terms of conjugacy: if the geodesic flows on two compact Riemannian manifolds are conjugate, then are the manifolds isometric?

\section{REFERENCES}

[1] Arnold, V. I. and A. Avez, Ergodic problems of classical mechanics. Translated from the French by A. Avez. W. A. Benjamin, Inc., New York-Amsterdam 1968.

[2] Besson, G., Courtois, G. and S. Gallot, Entropies et rigidités des espaces localement symétriques de courbure strictement négative, Geometric And Functional Analysis 5 (1995), 731799.

[3] Besson, G., Courtois, G. and S. Gallot, Minimal entropy and Mostow's rigidity theorems. Ergodic Theory Dynam. Systems 16 (1996), no. 4, 623649.

[4] W. Ballman, M. Gromov and V. Schroeder, Manifolds of nonpositive curvature. Birkhäuser, Boston 1985.

[5] Bonahon, Francis, The geometry of Teichmüller space via geodesic currents. Invent. Math. 92 (1988), no. 1, 139162.

[6] M. Bridson and A. Haefliger, Metric spaces of non-positive curvature. SpringerVerlag, Berlin, 1999.

[7] Burns, K. and Katok, A., Manifolds with nonpositive curvature, Ergodic Theory Dynam. Systems, 5 (1985), 307-317.

[8] Croke, Christopher B, Rigidity for surfaces of nonpositive curvature. Comment. Math. Helv. 65 (1990), no. 1, 150169.

[9] Croke, Christopher B, Rigidity theorems in Riemannian geometry. Geometric methods in inverse problems and PDE control, 4772, IMA Vol. Math. Appl., 137, Springer, New York, 2004.

[10] Croke, Christopher and Bruce Kleiner, Conjugacy and rigidity for manifolds with a parallel vector field. J. Differential Geom. 39 (1994), no. 3, 659680.

[11] Croke, Christopher B. and Vladimir A. Sharafutdinov, Spectral rigidity of a compact negatively curved manifold. Topology 37 (1998), no. 6, 12651273. 
[12] de la Llave, R. and R. Moriyón, Invariants for smooth conjugacy of hyperbolic dynamical systems. IV. Comm. Math. Phys. 116 (1988), no. 2, 185192.

[13] Farrell, F. Thomas and Pedro Ontaneda, On the topology of the space of negatively curved metrics. J. Differential Geom. 86 (2010), no. 2, 273301.

[14] Feldman, J. and D. Ornstein, Semirigidity of horocycle flows over compact surfaces of variable negative curvature. Ergodic Theory Dynam. Systems 7 (1987), no. 1, 4972.

[15] Furman, Alex, Coarse-geometric perspective on negatively curved manifolds and groups. (English summary) Rigidity in dynamics and geometry (Cambridge, 2000), 149166, Springer, Berlin, 2002.

[16] Gordon, Carolyn, Isospectral closed Riemannian manifolds which are not locally isometric. J. Differential Geom. 37 (1993), no. 3, 639649.

[17] Guillemin, V. and D. Kazhdan, Some inverse spectral results for negatively curved 2-manifolds. Topology 19 (1980), no. 3, 301312.

[18] Hamenstädt, U., Cocycles, symplectic structures and intersection. Geom. Funct. Anal. 9 (1999), no. 1, 90140.

[19] Hamilton, R.S., The Ricci flow on surfaces, in Mathematics and General Relativity (Santa Cruz, CA, 1986) (J. A. Isenberg, ed.), Contemp. Math. 71, Amer. Math. Soc., Providence, RI, 1988, pp. 237262.

[20] Hirsch, M. W.; Pugh, C. C.; Shub, M. Invariant manifolds. Lecture Notes in Mathematics, Vol. 583. Springer-Verlag, Berlin-New York, 1977.

[21] A. Katok and B. Hasselblatt, Introduction to the modern theory of dynamical systems. Encyclopedia of Mathematics and its Applications, 54. Cambridge University Press, Cambridge, 1995.

[22] Klingenberg, Wilhelm, Riemannian geometry. Second edition. de Gruyter Studies in Mathematics, 1. Walter de Gruyter \& Co., Berlin, 1995.

[23] Labourie, François, What is ... a cross ratio? Notices Amer. Math. Soc.55 (2008), no. 10, 12341235.

[24] Ledrappier, François, Structure au bord des variétés à courbure négative. (French) Séminaire de Théorie Spectrale et Géométrie, No. 13, Année 19941995, 97122, Sémin. Théor. Spectr. Géom., 13, Univ. Grenoble I, Saint-Martin-d'Hères, 1995.

[25] McMullen, C. Hyperbolic manifolds, discrete groups and ergodic theory Course notes, http://www.math.harvard.edu/ ctm/home/text/class/berkeley/277/96 /course/course.pdf

[26] McMullen, C. Teichmüller Theory Notes, Course notes, http://www.math.harvard.edu/ ctm/home/text/class/harvard/275/05/html /home/course/course.pdf

[27] Otal, Jean-Pierre, Le spectre marqué des longueurs des surfaces à courbure négative. (French) [The marked spectrum of the lengths of surfaces with negative curvature] Ann. of Math. (2) 131 (1990), no. 1, 151162.

[28] Santaló, Luis A. Integral geometry and geometric probability. Second edition. Cambridge Mathematical Library. Cambridge University Press, Cambridge, 2004.

[29] Shub, Michael; Sullivan, Dennis, Expanding endomorphisms of the circle revisited. Ergodic Theory Dynam. Systems 5 (1985), no. 2, 285289.

[30] Sunada, Toshikazu Riemannian coverings and isospectral manifolds. Ann. of Math. (2) 121 (1985), no. 1, 169186.

[31] Spatzier, R. J., An invitation to rigidity theory. Modern dynamical systems and applications, 211231, Cambridge Univ. Press, Cambridge, 2004.

[32] Vignéras, Marie-France, Variétés riemanniennes isospectrales et non isométriques. (French) Ann. of Math. (2) 112 (1980), no. 1, 2132. 